\title{
Self-induced decoherence in dense neutrino gases
}

\author{
Georg G. Raffelt ${ }^{1}$ and Günter Sigl ${ }^{2}$ \\ ${ }^{1}$ Max-Planck-Institut für Physik (Werner-Heisenberg-Institut), Föhringer Ring 6, 80805 München, Germany \\ ${ }^{2} \mathrm{APC}^{*}$ (AstroParticules et Cosmologie), 10, rue Alice Domon et Léonie Duquet, 75205 Paris Cedex 13, France
}

(Dated: 22 January 2007)

\begin{abstract}
Dense neutrino gases exhibit collective oscillations where "self-maintained coherence" is a characteristic feature, i.e., neutrinos of different energies oscillate with the same frequency. In a nonisotropic gas, however, the flux term of the neutrino-neutrino interaction has the opposite effect of causing kinematical decoherence of neutrinos propagating in different directions, an effect that is at the origin of the "multi-angle behavior" of neutrinos streaming off a supernova core. We cast the equations of motion in a form where the role of the flux term is manifest. We study in detail the symmetric case of equal neutrino and antineutrino densities where the evolution consists of collective pair conversions ("bipolar oscillations"). A gas of this sort is unstable in that an infinitesimal anisotropy is enough to trigger a run-away towards flavor equipartition. The "self-maintained coherence" of a perfectly isotropic gas gives way to "self-induced decoherence."
\end{abstract}

PACS numbers: 14.60.Pq, 97.60.Bw

\section{INTRODUCTION}

Neutrino oscillations between two flavors in vacuum are governed by the frequency

$$
\omega=\frac{\Delta m^{2}}{2 E}
$$

where $E$ is the energy of a given mode. Therefore, if the energy spectrum is broad, the energy dependence of the oscillation frequency quickly leads to kinematical decoherence, i.e., along a neutrino beam the overall flavor content quickly approaches an average value.

The situation changes radically when neutrinos themselves provide a significant refractive effect, leading to collective oscillation modes [1, 2, 3, 4, 5, 6, 7, 8, 9, 10, 11, 12] that can be of practical interest in the early universe 13, 14, 15, 16 or in core-collapse supernovae [17, 18, 19, 20, 21, 22, 23, 24, 25, 26]. Defining the parameter

$$
\mu=\sqrt{2} G_{\mathrm{F}} n_{\nu},
$$

the neutrino gas is "dense" when $\omega \lesssim \mu$, i.e., when a typical neutrino-neutrino interaction energy exceeds the energy corresponding to the vacuum oscillation frequency. When this condition is satisfied, collective effects are important, even if the ordinary matter effect is much larger than that from the neutrino-neutrino interaction [23, 26]. One characteristic feature of collective oscillations is the phenomenon of "self-maintained coherence" [6], meaning that all modes oscillate with the same frequency even though the energy spectrum may be broad.

It was recently stressed, however, that this is not the complete story [11, 24]. Perhaps the most interesting case for collective effects is provided by neutrinos

*UMR 7164 (CNRS, Université Paris 7, CEA, Observatoire de
Paris) streaming off a supernova core, a situation that is far from isotropic. The current-current nature of the weakinteraction Hamiltonian implies that the interaction energy between particles of momenta $\mathbf{p}$ and $\mathbf{q}$ is proportional to $\left(1-\mathbf{v}_{\mathbf{p}} \cdot \mathbf{v}_{\mathbf{q}}\right)$ where $\mathbf{v}_{\mathbf{p}}=\mathbf{p} / E$ is the velocity. If the medium is isotropic, the $\mathbf{v}_{\mathbf{p}} \cdot \mathbf{v}_{\mathbf{q}}$ term averages to zero, but if there is a net current, test particles moving in different directions experience different refractive effects. Therefore, neutrinos moving in a background with a net current will decohere between different directions of motion, even if the energy spectrum is monochromatic.

To avoid confusion about terminology we stress that we always mean "kinematical decoherence" when we say "decoherence." Different modes oscillate differently, leading to "de-phasing" and thus to the depolarization of the overall ensemble. If we use the common language of polarization vectors $\mathbf{P}_{\mathbf{p}}$ for each mode $\mathbf{p}$, then the length of each $\mathbf{P}_{\mathbf{p}}$ is conserved, whereas the length of the overall polarization vector $\mathbf{P}=\sum \mathbf{P}_{\mathbf{p}}$ shrinks (kinematical decoherence) or is conserved (kinematical coherence). The effect of dynamical decoherence, caused by collisions among the neutrinos or with a thermal background medium, is that each individual polarization vector $\mathbf{P}_{\mathbf{p}}$ shrinks, i.e., neutrinos in individual modes cannot be represented as pure states. This effect, relevant for open quantum systems, does not occur in our case where oscillations are the only form of evolution.

The multi-angle decoherence effect becomes nontrivial in the most interesting case when the "background current" consists of the neutrinos themselves as for neutrinos streaming off a supernova core. Numerical examples reveal significant decoherence effects, but on the other hand they also show collective modes very similar to the isotropic ("single-angle") case [24]. The overall behavior is determined by a complicated interplay between the collective evolution and decoherence.

Rather than trying to understand the supernova case in its full complexity, we here take the opposite approach and study the simplest example that shows de- 
coherence caused by the neutrino-neutrino multi-angle effect. Therefore, we consider a dense neutrino gas that is monochromatic, symmetric (equal $\nu$ and $\bar{\nu}$ densities), and homogeneous, but not isotropic. Even this simple model has a rich phenomenology that helps one to develop a better understanding of the full problem. On the other hand, it is simple enough that the most puzzling aspects of its behavior are analytically tractable.

A symmetric $\nu \bar{\nu}$ gas oscillates in the "bipolar mode" where pairs of neutrinos of a given flavor coherently oscillate into the other flavor and back with the "bipolar frequency" [6, 8, 23, 26]

$$
\kappa=\sqrt{2 \omega \mu} .
$$

As an example we consider a dense neutrino gas initially consisting of $\nu_{e} \bar{\nu}_{e}$ and assume that the mixing angle $\Theta$ with another flavor is small, a situation that could represent 13-oscillations. We characterize the overall flavor content of the ensemble in the usual way by a polarization vector where the positive $z$-direction corresponds to the $\nu_{e}$-flavor whereas the mass direction is defined by a unit vector that we call $\mathbf{B}$. It is tilted against the $z$-direction by $2 \Theta$ and we always choose it to lie in the $x$-z-plane with $B_{z}<0$ for the normal hierarchy and $B_{z}>0$ for the inverted hierarchy.

In vacuum, the polarization vectors precess around $\mathbf{B}$ with frequency $\omega$. In a dense neutrino gas with $\Theta \ll 1$, their motion is largely confined to the $x$ - $z$-plane where they perform pendular motions with frequency $\kappa$ and where $\mathbf{- B}$ is the "force direction." The maximum excursion is $2 \Theta$, indicated by the dotted lines in Fig. 1 where we show the trajectory in the $x$ - $z$-plane. For the normal hierarchy, the pendulum swings between the dotted lines. For the inverted hierarchy (bottom), it performs almost full-circle oscillations as indicated by the diamonds.

If the gas is not isotropic, the picture changes completely. Taking the "half-isotropic" case as a generic example where only one half-space of momentum modes is occupied (neutrinos streaming off a plane surface), we show in Fig. 1 the trajectory of the average polarization vector. For both hierarchies, its length shrinks to zero so that the final state of the ensemble is an equal mixture of both flavors. In other words, the ensemble quickly decoheres, independently of the mass hierarchy. This behavior is perhaps intuitive because we have assumed a large neutrino current.

However, this effect is self-accelerating in that it is triggered even by the smallest nonzero anisotropy. Whereas for perfect isotropy the neutrino-neutrino interaction provides for self-maintained coherence between modes of different energy, even an infinitesimal anisotropy has the opposite effect of causing self-induced decoherence between modes with different propagation directions. The average flavor content of the ensemble always approaches an equal mixture of both flavors. This instability is a nonlinear effect caused by the neutrino-neutrino interaction. In other words, the pendular motion of the isotropic ensemble is an unstable limit cycle, whereas the equal flavor

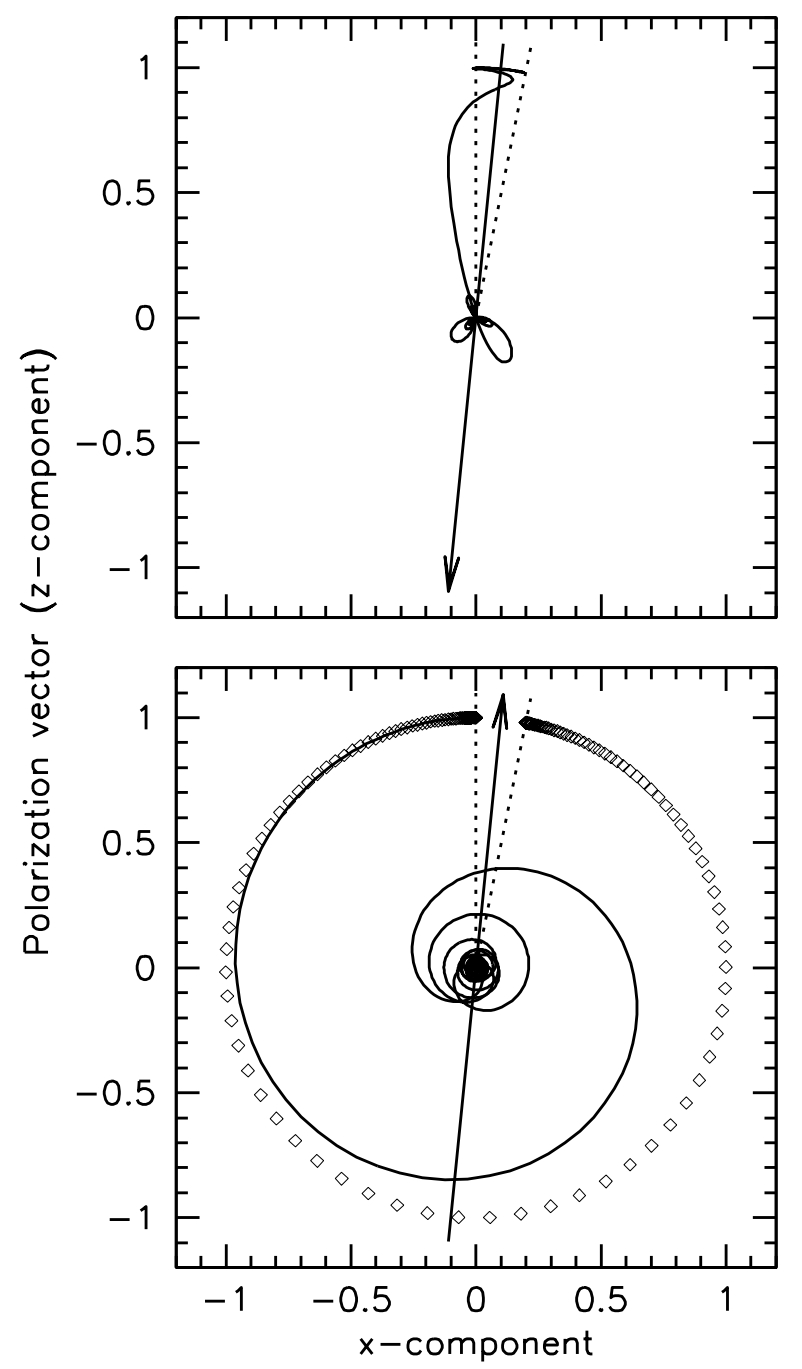

FIG. 1: Trajectory of the overall polarization vector in the "half-isotropic case" for the normal hierarchy (top) and inverted hierarchy (bottom) with $\sin 2 \Theta=0.1$. The arrow is the $\mathbf{B}$ direction. In the isotropic case (no decoherence), the dotted lines represent the largest pendulum excursion of $2 \Theta$ on either side of $\mathbf{B}$. The diamonds in the lower panel represent the pendular motion in time intervals of $0.01(2 \pi / \kappa)$.

mixture is a stable fixed point.

Our goal is to explain this behavior. As a first step we formulate in Sect. II the equations of motion for the multi-angle system in terms of the multipole components of the neutrino angular distribution. The special role of the flux (the dipole term of the angular distribution) becomes manifest. We begin with the full $N$-flavor problem and then consider two flavors in terms of polarization vectors. In Sect. III we study the role of a large anisotropy in causing decoherence. Our main result is developed in Sect.IV where we show that a $\nu \bar{\nu}$ gas is unstable in flavor space and always decoheres if there is an infinitesimally small initial anisotropy. We conclude in Sec. V with a summary and outlook. 


\section{MULTI-ANGLE EQUATIONS OF MOTION}

\section{A. General equations of motion}

A statistical ensemble of unmixed neutrinos is represented by the occupation numbers $f_{\mathbf{p}}=\left\langle a_{\mathbf{p}}^{\dagger} a_{\mathbf{p}}\right\rangle$ for each momentum mode $\mathbf{p}$, where $a_{\mathbf{p}}^{\dagger}$ and $a_{\mathbf{p}}$ are the relevant creation and annihilation operators and $\langle\ldots\rangle$ is the expectation value. The corresponding expression for antineutrinos is $\bar{f}_{\mathbf{p}}=\left\langle\bar{a}_{\mathbf{p}}^{\dagger} \bar{a}_{\mathbf{p}}\right\rangle$, where here and henceforth overbarred quantities refer to antiparticles.

For several flavors, the occupation numbers are generalized to "matrices of densities" in flavor space [30, 31, $32,33]$

$$
\left(\varrho_{\mathbf{p}}\right)_{i j}=\left\langle a_{j}^{\dagger} a_{i}\right\rangle_{\mathbf{p}} \quad \text { and } \quad\left(\bar{\varrho}_{\mathbf{p}}\right)_{i j}=\left\langle\bar{a}_{i}^{\dagger} \bar{a}_{j}\right\rangle_{\mathbf{p}}
$$

that really are matrices of occupation numbers. The re- versed order of the flavor indices $i$ and $j$ in the r.h.s. for antineutrinos ensures that under a flavor transformation, $\varrho_{\mathbf{p}} \rightarrow U \varrho_{\mathbf{p}} U^{\dagger}$, antineutrinos transform in the same way, $\bar{\varrho}_{\mathbf{p}} \rightarrow U \bar{\varrho}_{\mathbf{p}} U^{\dagger}$. Sums and differences of $\varrho_{\mathbf{p}}$ and $\bar{\varrho}_{\mathbf{p}}$ then transform consistently. For the seemingly intuitive equal order of flavor indices that is almost universally used in the literature, $\varrho_{\mathbf{p}}, \bar{\varrho}_{\mathbf{p}}, \varrho_{\mathbf{p}}^{*}$ and $\bar{\varrho}_{\mathbf{p}}^{*}$ all appear in the equations instead of $\varrho_{\mathbf{p}}$ and $\bar{\varrho}_{\mathbf{p}}$ alone.

Analogous matrices $\left(\ell_{\mathbf{p}}\right)_{i j}$ can be defined for the charged leptons.

We assume that the neutrino ensemble is completely described by these one-particle occupation number matrices, i.e., that genuine many-body effects play no role [27, 28, 29]. In this case flavor oscillations of an ensemble of neutrinos and antineutrinos are described by an equation of motion for each mode [30, 31, 32, 33]

$$
\begin{aligned}
& \mathrm{i} \partial_{t} \varrho_{\mathbf{p}}=+\left[\Omega_{\mathbf{p}}, \varrho_{\mathbf{p}}\right]+\sqrt{2} G_{\mathrm{F}}\left[\int \frac{d^{3} \mathbf{q}}{(2 \pi)^{3}}\left(\ell_{\mathbf{q}}-\bar{\ell}_{\mathbf{q}}+\varrho_{\mathbf{q}}-\bar{\varrho}_{\mathbf{q}}\right)\left(1-\mathbf{v}_{\mathbf{q}} \cdot \mathbf{v}_{\mathbf{p}}\right), \varrho_{\mathbf{p}}\right], \\
& \mathrm{i} \partial_{t} \bar{\varrho}_{\mathbf{p}}=-\left[\Omega_{\mathbf{p}}, \bar{\varrho}_{\mathbf{p}}\right]+\sqrt{2} G_{\mathrm{F}}\left[\int \frac{d^{3} \mathbf{q}}{(2 \pi)^{3}}\left(\ell_{\mathbf{q}}-\bar{\ell}_{\mathbf{q}}+\varrho_{\mathbf{q}}-\bar{\varrho}_{\mathbf{q}}\right)\left(1-\mathbf{v}_{\mathbf{q}} \cdot \mathbf{v}_{\mathbf{p}}\right), \bar{\varrho}_{\mathbf{p}}\right],
\end{aligned}
$$

where $[\cdot, \cdot]$ is a commutator and $G_{\mathrm{F}}$ is the Fermi constant. For ultrarelativistic neutrinos, the matrix of vacuum oscillation frequencies, expressed in the mass basis, is $\Omega_{\mathbf{p}}=\operatorname{diag}\left(m_{1}^{2}, m_{2}^{2}, m_{3}^{2}\right) / 2 p$ with $p=|\mathbf{p}|$. Further, $\mathbf{v}_{\mathbf{p}}=\mathbf{p} / E_{\mathbf{p}}$ is the velocity of a particle (neutrino or charged lepton) with momentum $\mathbf{p}$.

The total conserved energy of the neutrino ensemble is an important quantity for understanding its evolution [23, 26]. We find for the energy density

$$
\begin{array}{r}
\varepsilon=\operatorname{Tr}\left\{\int \frac{d^{3} \mathbf{p}}{(2 \pi)^{3}} \Omega_{\mathbf{p}}\left(\varrho_{\mathbf{p}}+\bar{\varrho}_{\mathbf{p}}\right)+\sqrt{2} G_{\mathrm{F}} \int \frac{d^{3} \mathbf{p}}{(2 \pi)^{3}} \int \frac{d^{3} \mathbf{q}}{(2 \pi)^{3}}\left(\ell_{\mathbf{q}}-\bar{\ell}_{\mathbf{q}}\right)\left(\varrho_{\mathbf{p}}-\bar{\varrho}_{\mathbf{p}}\right)\left(1-\mathbf{v}_{\mathbf{q}} \cdot \mathbf{v}_{\mathbf{p}}\right)\right. \\
\left.+\frac{\sqrt{2} G_{\mathrm{F}}}{2}\left[\int \frac{d^{3} \mathbf{p}}{(2 \pi)^{3}}\left(\varrho_{\mathbf{p}}-\bar{\varrho}_{\mathbf{p}}\right)\right]^{2}-\frac{\sqrt{2} G_{\mathrm{F}}}{2}\left[\int \frac{d^{3} \mathbf{p}}{(2 \pi)^{3}}\left(\varrho_{\mathbf{p}}-\bar{\varrho}_{\mathbf{p}}\right) \mathbf{v}_{\mathbf{p}}\right]^{2}\right\} .
\end{array}
$$

This quantity actually represents the energy shift caused by the neutrino masses and by neutrino interactions. It is straightfoward to show that indeed $\dot{\varepsilon}=0$ by taking the time derivative on the r.h.s. of Eq. (6), inserting the equations of motion Eq. (5), and using cyclic permutations of matrices under the trace.

For completeness we mention that one can also define the entropy density 32]

$$
\begin{gathered}
s=-\int \frac{d^{3} \mathbf{p}}{(2 \pi)^{3}} \operatorname{Tr}\left[\varrho_{\mathbf{p}} \ln \left(\varrho_{\mathbf{p}}\right)+\left(1-\varrho_{\mathbf{p}}\right) \ln \left(1-\varrho_{\mathbf{p}}\right)\right. \\
\left.+\bar{\varrho}_{\mathbf{p}} \ln \left(\bar{\varrho}_{\mathbf{p}}\right)+\left(1-\bar{\varrho}_{\mathbf{p}}\right) \ln \left(1-\bar{\varrho}_{\mathbf{p}}\right)\right] .
\end{gathered}
$$

This expression is well defined because $\varrho_{\mathbf{p}}$ and $1-\varrho_{\mathbf{p}}$ are positive semi-definite matrices. In our case where the equation of motion for each $\varrho_{\mathbf{p}}$ is of the form i $\partial_{t} \varrho_{\mathbf{p}}=$ $\left[H, \varrho_{\mathbf{p}}\right]$, the entropy density is conserved. Oscillations alone do not lead to a loss of information. Kinematical decoherence does not lead to an increase of entropy.

\section{B. Axial symmetry}

Since we wish to study the simplest example that shows nontrivial multi-angle effects, we now restrict the neutrino energy distribution to be monochromatic and the geometry to axial symmetry. We then integrate the matrices of densities over all variables except $u=\cos \theta_{\mathbf{p}}$ where the angle is relative to the direction of symmetry. Therefore, we consider the matrices

$$
\varrho_{u}=\frac{1}{n_{\nu}} \int \frac{d p p^{2} d \varphi}{(2 \pi)^{3}} \varrho_{\mathbf{p}}
$$


implying the normalization

$$
\varrho_{0} \equiv \frac{1}{n_{\nu}} \int \frac{d^{3} \mathbf{p}}{(2 \pi)^{3}} \varrho_{\mathbf{p}}=\int_{-1}^{+1} d u \varrho_{u} .
$$

For convenience we have arbitrarily normalized the $\varrho_{u}$ matrices to the neutrino density.

We further define the matrix representing the particle flux along the direction of symmetry,

$$
\varrho_{1} \equiv \int_{-1}^{+1} d u \varrho_{u} v_{u}
$$

where $v_{u}$ is the velocity along the symmetry direction. For relativistic particles $v_{u}=\cos \theta_{u}=u$.

For charged leptons we proceed in the same way, except that we normalize to the electron density $n_{e}$.

We denote with $\Omega$ the matrix of vacuum oscillation frequencies for our fixed neutrino energy $E$. The equations of motion then simplify to

$$
\begin{aligned}
\mathrm{i} \dot{\varrho}_{u}=\left[\Omega, \varrho_{u}\right] & +\left[\lambda(\ell-\bar{\ell})_{0}+\mu(\varrho-\bar{\varrho})_{0}, \varrho_{u}\right] \\
& -\left[\lambda(\ell-\bar{\ell})_{1}+\mu(\varrho-\bar{\varrho})_{1}, u \varrho_{u}\right]
\end{aligned}
$$

where $\lambda=\sqrt{2} G_{\mathrm{F}} n_{e}$. The same equation applies for $\bar{\rho}_{u}$ except for a sign change of the vacuum oscillation term.

Evidently the isotropic part of the medium (index 0) affects all modes in the same way and is ultimately responsible for the phenomenon of self-maintained coherence. On the other hand, the flux term (index 1) involves a factor $u=\cos \theta$ for every mode $u$. Even in the absence of a neutrino-neutrino term, a charged-lepton flux alone causes a trivial multi-angle decoherence effect.

The conserved energy of the axially symmetric neutrino ensemble is

$$
\begin{aligned}
E= & \operatorname{Tr}\left[\Omega\left(\varrho_{0}+\bar{\varrho}_{0}\right)\right] \\
& +\lambda \operatorname{Tr}\left[(\ell-\bar{\ell})_{0}(\varrho-\bar{\varrho})_{0}-(\ell-\bar{\ell})_{1}(\varrho-\bar{\varrho})_{1}\right] \\
& +\frac{\mu}{2} \operatorname{Tr}\left[(\varrho-\bar{\varrho})_{0}^{2}-(\varrho-\bar{\varrho})_{1}^{2}\right] .
\end{aligned}
$$

Because of our normalization of the $\varrho_{u}$ matrices the quantity $E=\varepsilon / n_{\nu}$ is the energy per $\nu$.

\section{Expansion in Legendre polynomials}

The structure of these equations becomes more transparent if we expand the angular dependence of the matrices in Legendre polynomials. (Had we not assumed axial symmetry, spherical harmonics would be the appropriate basis.) The first few Legendre polynomials are

$$
\begin{aligned}
& L_{0}(u)=1, \\
& L_{1}(u)=u, \\
& L_{2}(u)=\frac{1}{2}\left(3 u^{2}-1\right) .
\end{aligned}
$$

We thus define

$$
\varrho_{n}=\int_{-1}^{+1} d u \varrho_{u} L_{n}(u)
$$

The normalisation

$$
\int_{-1}^{+1} d u L_{m}(u) L_{n}(u)=\frac{2}{2 n+1} \delta_{m n}
$$

implies that the original function is

$$
\varrho_{u}=\sum_{n=0}^{\infty}\left(n+\frac{1}{2}\right) \varrho_{n} L_{n}(u) .
$$

The previously defined overall density $\varrho_{0}$ and the flux $\varrho_{1}$ are but the zeroth and first case of Eq. (14).

Multiplying both sides of Eq. (11) with $L_{n}(u)$ and integrating over $d u$ leads to the equation of motion

$$
\begin{aligned}
\mathrm{i} \dot{\varrho}_{n}= & {\left[\Omega, \varrho_{n}\right]+\mu\left[(\varrho-\bar{\varrho})_{0}, \varrho_{n}\right] } \\
& -\mu\left[(\varrho-\bar{\varrho})_{1}, \int_{-1}^{+1} d u u \varrho_{u} L_{n}(u)\right] .
\end{aligned}
$$

Here and henceforth we no longer show the ordinary matter term. Its structure is similar to the neutrino-neutrino term so that it is easily reinstated.

With the expansion Eq. (16) the remaining integral is

$$
\begin{aligned}
& \int_{-1}^{+1} d u u \varrho_{u} L_{n}(u) \\
& \quad=\sum_{m=0}^{\infty}\left(m+\frac{1}{2}\right) \varrho_{m} \int_{-1}^{+1} d u u L_{m}(u) L_{n}(u) .
\end{aligned}
$$

We use the "dipole matrix element" of the Legendre polynomials

$$
\begin{aligned}
\int_{-1}^{+1} d u u L_{m}(u) L_{n}(u) & =\frac{2(m+1)}{(2 m+1)(2 m+3)} \delta_{m+1, n} \\
& +\frac{2 m}{(2 m-1)(2 m+1)} \delta_{m-1, n}
\end{aligned}
$$

With this result the equations of motion are

$$
\begin{aligned}
\mathrm{i} \dot{\varrho}_{n}= & {\left[\Omega, \varrho_{n}\right]+\mu\left[(\varrho-\bar{\varrho})_{0}, \varrho_{n}\right] } \\
& -\frac{\mu}{2}\left[(\varrho-\bar{\varrho})_{1},\left(a_{n} \varrho_{n-1}+b_{n} \varrho_{n+1}\right)\right],
\end{aligned}
$$

where

$$
\begin{aligned}
& a_{n}=\frac{2 n}{2 n+1}=1-\frac{1}{2 n+1} \\
& b_{n}=\frac{2(n+1)}{2 n+1}=1+\frac{1}{2 n+1} .
\end{aligned}
$$

Note that the equation for $\varrho_{0}$ is consistent even though the quantity $\varrho_{-1}$ is not defined because the coefficient $a_{n}=n /(2 n+1)=0$ for $n=0$.

The equations of motion Eq. (20) together with the corresponding equations for $\bar{\varrho}_{n}$ (sign change for the vacuum term) form a closed set of equations. If we also include the ordinary matter term they are equivalent to Eq. (11) for the momentum-space matrices $\varrho_{u}$. 
One important difference is that $\operatorname{Tr}\left(\varrho_{u}^{2}\right)$ is conserved, reflecting the absence of decoherence for individual momentum modes, whereas $\operatorname{Tr}\left(\varrho_{n}^{2}\right)$ is not in general conserved, reflecting the effect of kinematical decoherence. We note that in general $\partial_{t} \operatorname{Tr}\left(\mathrm{A}^{2}\right)=0$ when the equation of motion is of the form $i \partial_{t} A=[A, H]$. This is the case for $\varrho_{u}$, but not for the multipole matrices $\varrho_{n}$.

Of course, in a numerical implementation the series $\varrho_{n}$ has to be truncated at some value $n_{\max }$, leading to limited angular resolution. This is analogous to the coarse graining of phase space required for the $\rho_{u}$ where one needs to use discrete angular bins of nonzero width.

The equations of motion for the flux terms are special in that they involve one power of $\varrho_{1}$ or $\bar{\varrho}_{1}$ in each term of the equation. Therefore, if initially there is no flux term $\left(\varrho_{1}=\bar{\varrho}_{1}=0\right)$, none will develop. In this case the equations for $\varrho_{0}$ and $\bar{\varrho}_{0}$ form a closed set, describing the dynamics of the "flavor pendulum" studied in Ref. [26]. In addition, the higher multipoles $\varrho_{n}$ with $n \geq 2$, if initially nonzero, will simply oscillate under the action of the vacuum term and of the density term $(\varrho-\bar{\varrho})_{0}$.

\section{Diffusion equation in multipole space}

The interpretation of the equations of motion in multipole space becomes clearer if we observe that for some function $f(x)$ one has to second order

$$
\begin{gathered}
f(x+\Delta x)=f(x)+f^{\prime}(x) \Delta x+\frac{1}{2} f^{\prime \prime}(x) \Delta x^{2}, \\
f(x-\Delta x)=f(x)-f^{\prime}(x) \Delta x+\frac{1}{2} f^{\prime \prime}(x) \Delta x^{2} .
\end{gathered}
$$

Taking the sum and difference of these equations provides

$$
\begin{aligned}
& f(x+\Delta x)+f(x-\Delta x)=2 f(x)+f^{\prime \prime}(x) \Delta x^{2}, \\
& f(x+\Delta x)-f(x-\Delta x)=2 f^{\prime}(x) \Delta x .
\end{aligned}
$$

With Eq. (21) we may write

$$
a_{n} \varrho_{n-1}+b_{n} \varrho_{n+1}=2 \varrho_{n}+\frac{2}{2 n+1} \varrho_{n}^{\prime} \Delta n+\varrho_{n}^{\prime \prime} \Delta n^{2},
$$

where we interpret a prime as a derivative with respect to $n$ that is now viewed as a continuous variable. If we further observe that in the discrete equation $\Delta n=1$ we may write Eq. (20) in the continuous form

$$
\begin{aligned}
\mathrm{i} \dot{\varrho}_{n}=\left[\Omega, \varrho_{n}\right] & +\mu\left[(\varrho-\bar{\varrho})_{0}-(\varrho-\bar{\varrho})_{1}, \varrho_{n}\right] \\
-\mu & {\left[(\varrho-\bar{\varrho})_{1},\left(\frac{\varrho_{n}^{\prime}}{2 n+1}+\frac{\varrho_{n}^{\prime \prime}}{2}\right)\right] . }
\end{aligned}
$$

Therefore, the lowest multipole (monopole) is equivalent to the pendulum in flavor space [26], the high multipoles are akin to a continuous medium carrying flavor waves. Equation (25) is a diffusion equation. The periodic excitation caused by the flavor pendulum then diffuses to higher multipoles (smaller scales). The flavor waves as a function of neutrino direction visible in numerical simulations [25] are the result of this process.

\section{E. Particle vs. lepton number}

In the equations of motion the difference of the particle and antiparticle densities, $\varrho_{\mathbf{p}}-\bar{\varrho}_{\mathbf{p}}$, plays a special role in that it is this matrix of net lepton number densities that plays the role of a potential for the other neutrino modes. As noted by several authors, it is sometimes useful to write the equations of motion in terms of the sum and difference of $\varrho_{\mathbf{p}}$ and $\bar{\varrho}_{\mathbf{p}}$ instead of these matrices themselves. We therefore define the matrix of particle densities (sum S) and the matrix of lepton number densities (difference $\mathrm{D}$ ) by virtue of

$$
\begin{aligned}
& \mathrm{S}_{\mathbf{p}}=\varrho_{\mathbf{p}}+\bar{\varrho}_{\mathbf{p}}, \\
& \mathrm{D}_{\mathbf{p}}=\varrho_{\mathbf{p}}-\bar{\varrho}_{\mathbf{p}} .
\end{aligned}
$$

Analogous definitions apply to the case of axial symmetry, $\mathrm{S}_{u}$ and $\mathrm{D}_{u}$, and their multipole expansion $\mathrm{S}_{n}$ and $\mathrm{D}_{n}$.

The equations of motion for the particle and lepton number matrices are found by adding and subtracting the two lines of Eq. (5) and the equivalent equations for axial symmetry or the multipoles. We find explicitly for the multipoles

$$
\begin{aligned}
i \dot{S}_{n} & =\left[\Omega, \mathrm{D}_{n}\right]+\mu\left[\mathrm{D}_{0}, \mathrm{~S}_{n}\right]-\mu\left[\mathrm{D}_{1}, \frac{a_{n} \mathrm{~S}_{n-1}+b_{n} \mathrm{~S}_{n+1}}{2}\right], \\
\mathrm{i} \dot{\mathrm{D}}_{n} & =\left[\Omega, \mathrm{S}_{n}\right]+\mu\left[\mathrm{D}_{0}, \mathrm{D}_{n}\right]-\mu\left[\mathrm{D}_{1}, \frac{a_{n} \mathrm{D}_{n-1}+b_{n} \mathrm{D}_{n+1}}{2}\right] .
\end{aligned}
$$

One can easily restore the ordinary matter term because it has the same structure as the neutrino-neutrino term with the substitution $\mu \rightarrow \lambda$ and $\mathrm{D}_{0,1} \rightarrow \mathrm{L}_{0,1}$, the latter being the charged-lepton matrix of net lepton-number densities and the corresponding flux, respectively.

The commutator structure of the r.h.s. of Eq. (27) implies that its trace vanishes. Therefore, the particle and lepton numbers for all multipoles, $\operatorname{Tr}\left(\mathrm{S}_{n}\right)$ and $\operatorname{Tr}\left(\mathrm{D}_{n}\right)$, are conserved.

With the expressions Eq. (21) for $a_{n}$ and $b_{n}$ the lowestorder multipole equation is

$$
\begin{aligned}
\mathrm{i} \dot{\mathrm{S}}_{0} & =\left[\Omega, \mathrm{D}_{0}\right]+\mu\left[\mathrm{D}_{0}, \mathrm{~S}_{0}\right]-\mu\left[\mathrm{D}_{1}, \mathrm{~S}_{1}\right], \\
\mathrm{i} \dot{\mathrm{D}}_{0} & =\left[\Omega, \mathrm{S}_{0}\right] .
\end{aligned}
$$

One immediate consequence of the second equation is

$$
\partial_{t} \operatorname{Tr}\left(\Omega \mathrm{D}_{0}\right)=0,
$$

i.e., in the mass basis a certain combination of flavorlepton numbers is conserved.

The explicit equation for the flux term is

$$
\begin{aligned}
\mathrm{iS}_{1} & =\left[\Omega, \mathrm{D}_{1}\right]+\mu\left[\mathrm{D}_{0}, \mathrm{~S}_{1}\right]+\frac{\mu}{3}\left[\mathrm{~S}_{0}+2 \mathrm{~S}_{2}, \mathrm{D}_{1}\right], \\
\mathrm{i} \dot{\mathrm{D}}_{1} & =\left[\Omega, \mathrm{S}_{1}\right]+\frac{2 \mu}{3}\left[\mathrm{D}_{0}-\mathrm{D}_{2}, \mathrm{D}_{1}\right] .
\end{aligned}
$$

As stated earlier, these equations are special in that they involve one power of $S_{1}$ or $D_{1}$ in each term. Therefore, $S_{1}$ and $D_{1}$ vanish at all times if they vanish initially. 
In this case, the monopole terms (overall densities) form a closed set of equations. Defining

$$
\begin{aligned}
& \mathrm{Q}=\mathrm{S}_{0}-\frac{\Omega}{\mu}, \\
& \mathrm{D}=\mathrm{D}_{0},
\end{aligned}
$$

the equations of motion take on the simple form

$$
\begin{aligned}
& \mathrm{i} \dot{\mathrm{Q}}=\mu[\mathrm{D}, \mathrm{Q}], \\
& \mathrm{i} \dot{\mathrm{D}}=[\Omega, \mathrm{Q}] .
\end{aligned}
$$

Conserved quantities are $\operatorname{Tr}\left(Q^{2}\right), \operatorname{Tr}(D Q), \operatorname{Tr}(D \Omega)$, and $\operatorname{Tr}\left(\Omega \mathrm{Q}+\mu \mathrm{D}^{2} / 2\right)$. Up to a constant, the last quantity is the energy per neutrino. For two flavors, these simplifications are at the origin of the pendulum analogy [26] where $\operatorname{Tr}\left(\mathrm{Q}^{2}\right)$ corresponds to its length, $\operatorname{Tr}(\mathrm{DQ})$ its spin, and $\operatorname{Tr}(D \Omega)$ to the orbital angular momentum around the force direction (the mass direction in flavor space).

Returning to the general case with arbitrary initial conditions, the energy per neutrino is

$$
E=\operatorname{Tr}\left[\Omega \mathrm{S}_{0}+\frac{\mu}{2}\left(\mathrm{D}_{0}^{2}-\mathrm{D}_{1}^{2}\right)\right] .
$$

Energy conservation $\partial_{t} E=0$ is easily verified by using the equations of motion for $S_{0}, D_{0}$ and $D_{1}$.

\section{F. Two-flavor case}

In the two-flavor case where the $\varrho_{\mathbf{p}}$ are Hermitian $2 \times 2$ matrices, a representation in terms of polarization vectors is useful. A Hermitian $2 \times 2$ matrix $A$ is represented as

$$
\mathrm{A}=\frac{\operatorname{Tr}(\mathrm{A})+\mathbf{A} \cdot \boldsymbol{\sigma}}{2},
$$

where $\boldsymbol{\sigma}$ is the vector of Pauli matrices and $\mathbf{A}$ the polarization vector.

The commutation relations of the Pauli matrices imply that an equation of motion of the form

$$
\mathrm{i} \partial_{t} \mathrm{~A}=[\mathrm{B}, \mathrm{C}]
$$

is represented by

$$
\partial_{t} \operatorname{Tr}(\mathbf{A})=0 \quad \text { and } \quad \partial_{t} \mathbf{A}=\mathbf{B} \times \mathbf{C} .
$$

Moreover,

$$
\operatorname{Tr}(\mathrm{AB})=\frac{\operatorname{Tr}(\mathrm{A}) \operatorname{Tr}(\mathrm{B})+\mathbf{A} \cdot \mathbf{B}}{2}
$$

for any two matrices $\mathrm{A}$ and $\mathrm{B}$.

As usual, we denote with $\mathbf{P}_{\mathbf{p}}$ and $\overline{\mathbf{P}}_{\mathbf{p}}$ the polarization vectors representing $\varrho_{\mathbf{p}}$ and $\bar{\varrho}_{\mathbf{p}}$, respectively, and analogous expressions for $\varrho_{u} \rightarrow \mathbf{P}_{u}$ and $\varrho_{n} \rightarrow \mathbf{P}_{n}$. The particle and lepton-number matrices are represented by $\mathbf{S} \rightarrow \mathbf{S}$ and $\mathbf{D} \rightarrow \mathbf{D}$, in each case with the subscripts $\mathbf{p}, u$ or $n$ for the different variables. Finally, we represent the matrix of oscillation frequencies in the form

$$
\Omega=\frac{\operatorname{Tr}(\Omega)+\omega \mathbf{B} \cdot \boldsymbol{\sigma}}{2}
$$

where

$$
\operatorname{Tr}(\Omega)=\frac{m_{1}^{2}+m_{2}^{2}}{2 E} \quad \text { and } \quad \omega=\left|\frac{m_{1}^{2}-m_{2}^{2}}{2 E}\right| .
$$

We have defined the oscillation frequency $\omega$ as a positive number. The "magnetic field" is a unit vector that in the interaction basis is explicitly $\mathbf{B}=(\sin 2 \Theta, 0, \cos 2 \Theta)$ where $\Theta$ is the vacuum mixing angle. We have arbitrarily chosen its $y$-component to vanish, corresponding to a choice of overall phase of the mixing matrix. In the following, when we say that "the mixing angle is small," we mean that $|\sin 2 \Theta| \ll 1$. The sign of $B_{x}$ is physically irrelevant whereas $B_{z}<0$ represents the normal, $B_{z}>0$ the inverted hierarchy.

The equations of motion for the particle- and leptonnumber polarization vectors are explicitly

$$
\begin{aligned}
& \dot{\mathbf{S}}_{n}=\omega \mathbf{B} \times \mathbf{D}_{n}+\mu \mathbf{D}_{0} \times \mathbf{S}_{n}-\frac{\mu}{2} \mathbf{D}_{1} \times\left(a_{n} \mathbf{S}_{n-1}+b_{n} \mathbf{S}_{n+1}\right), \\
& \dot{\mathbf{D}}_{n}=\omega \mathbf{B} \times \mathbf{S}_{n}+\mu \mathbf{D}_{0} \times \mathbf{D}_{n}-\frac{\mu}{2} \mathbf{D}_{1} \times\left(a_{n} \mathbf{D}_{n-1}+b_{n} \mathbf{D}_{n+1}\right) .
\end{aligned}
$$

The two lowest-order equations are explicitly

$$
\begin{aligned}
\dot{\mathbf{S}}_{0} & =\omega \mathbf{B} \times \mathbf{D}_{0}+\mu \mathbf{D}_{0} \times \mathbf{S}_{0}-\mu \mathbf{D}_{1} \times \mathbf{S}_{1}, \\
\dot{\mathbf{D}}_{0} & =\omega \mathbf{B} \times \mathbf{S}_{0}
\end{aligned}
$$

and

$$
\begin{aligned}
\dot{\mathbf{S}}_{1} & =\omega \mathbf{B} \times \mathbf{D}_{1}+\mu \mathbf{D}_{0} \times \mathbf{S}_{1}+\frac{1}{3} \mu\left(\mathbf{S}_{0}+2 \mathbf{S}_{2}\right) \times \mathbf{D}_{1}, \\
\dot{\mathbf{D}}_{1} & =\omega \mathbf{B} \times \mathbf{S}_{1}+\frac{2}{3} \mu\left(\mathbf{D}_{0}-\mathbf{D}_{2}\right) \times \mathbf{D}_{1} .
\end{aligned}
$$


The conserved energy Eq. (33) becomes, with the help of Eq. (37),

$$
\begin{aligned}
E= & \frac{1}{2}\left\{\operatorname{Tr}(\Omega) \operatorname{Tr}\left(\mathrm{S}_{0}\right)+\omega \mathbf{B} \cdot \mathbf{S}_{0}\right. \\
& \left.+\frac{\mu}{2}\left[\operatorname{Tr}\left(\mathrm{D}_{0}\right)^{2}-\operatorname{Tr}\left(\mathrm{D}_{1}\right)^{2}+\mathbf{D}_{0}^{2}-\mathbf{D}_{1}^{2}\right]\right\} .
\end{aligned}
$$

We have already noted that the individual traces $\operatorname{Tr}\left(\mathrm{S}_{0}\right)$, $\operatorname{Tr}\left(D_{0}\right)$ and $\operatorname{Tr}\left(D_{1}\right)$ are conserved so that ${ }^{1}$

$$
E=\frac{1}{2}\left[\omega \mathbf{B} \cdot \mathbf{S}_{0}+\frac{\mu}{2}\left(\mathbf{D}_{0}^{2}-\mathbf{D}_{1}^{2}\right)\right]
$$

is the conserved energy per neutrino, up to an irrelevant constant. We use the notation

$$
E_{\omega}=\frac{\omega}{2}\left(\left|\mathbf{S}_{0}\right|+\mathbf{B} \cdot \mathbf{S}_{0}\right)
$$

for what is the "potential energy" in the pendulum analogy. Its minimum is chosen at zero. The "kinetic energy" (neutrino-neutrino interaction energy) is denoted by

$$
E_{\mu}=E_{0}+E_{1}=\frac{\mu}{4}\left(\mathbf{D}_{0}^{2}-\mathbf{D}_{1}^{2}\right)
$$

where we have introduced

$$
\begin{aligned}
& E_{0}=+\frac{\mu}{4} \mathbf{D}_{0}^{2}, \\
& E_{1}=-\frac{\mu}{4} \mathbf{D}_{1}^{2}
\end{aligned}
$$

for the monopole and dipole contributions.

\section{ROLE OF ANISOTROPY}

\section{A. Isotropic case}

In order to study the impact of kinematical decoherence in the simplest nontrivial case we study a homogeneous ensemble consisting of equal numbers of neutrinos and antineutrinos that are all initially in the electron flavor. Before turning to issues of decoherence, we briefly recall the evolution of the isotropic case. To this end we show in Fig. 2 in the upper panels the $z$-component of the overall polarization vector $\mathbf{S}_{0}=\mathbf{P}_{0}+\overline{\mathbf{P}}_{0}$ for the inverted and normal hierarchy. The $\nu_{e}$ or $\bar{\nu}_{e}$ survival probability is

$$
\operatorname{prob}\left(\nu_{e} \rightarrow \nu_{e}\right)=\operatorname{prob}\left(\bar{\nu}_{e} \rightarrow \bar{\nu}_{e}\right)=\frac{S_{0}^{z}+2}{4},
$$

because in our normalization $\left|\mathbf{S}_{0}\right|=2$. In the lower panels we show the corresponding energy components.

For the normal hierarchy (left) the motion is a smallexcursion harmonic oscillation with the bipolar frequency $\kappa$. We show one full cycle of oscillation. The

\footnotetext{
1 The overall factor $1 / 2$ is missing in the equivalent expression in our previous paper $[26$.
}

potential energy $E_{\omega}$ (solid line in the lower panel) is measured against the mass direction so that it is back at its maximum after a half-period as behooves an ordinary pendulum. The dashed line shows the "kinetic pendulum energy" $E_{\mu}$. The isotropy of the ensemble implies $E_{1}=0$ and thus $E_{\mu}=E_{0}$.

For the inverted hierarchy (right) the motion is that of an inverted oscillator. We show approximately one full cycle that for the chosen mixing angle lasts approximately 3 times the bipolar period $2 \pi / \kappa$. The polarization vector starts in the positive $z$ direction, swings almost one full circle and almost arrives back at the vertical direction (maximum in the middle of the plot), then swings back, reaching its original vertical position, and then starts over again. The maximum potential energy is $2 \omega$ because the energy is normalized to the total energy per $\nu$ and because there are equal numbers of neutrinos and antineutrinos, i.e., two particles per $\nu$.

Reducing the mixing angle has the effect of reducing the oscillation amplitude for the normal hierarchy, whereas it increases logarithmically the duration of the "plateau phases" for the inverted hierarchy. For quantitative discussions of the isotropic case in terms of the pendulum analogy see Ref. [26].

\section{B. Half-isotropic case}

Next we consider the same example, but assume a large degree of anisotropy where only one half-space of momentum modes is occupied. In Fig. 3 we show, in the upper panels, the evolution of $S_{0}^{z}$. Moreover, we show the length of the overall polarization vector, $\left|\mathbf{S}_{\mathbf{0}}\right|$, as a thin dashed line. Both in the normal hierarchy (left) and the inverted hierarchy (right), an equal mixture of the two flavors is quickly achieved. In the isotropic case the length of $\mathbf{P}_{0}$ and $\overline{\mathbf{P}}_{0}$ is conserved and that of $\mathbf{S}_{0}$ is approximately conserved, up to corrections of order $\omega / \mu \ll 1$. Here, the lengths of $\mathbf{P}_{0}, \overline{\mathbf{P}}_{0}$ and $\mathbf{S}_{0}$ shrink to zero, reflecting kinematical decoherence. Of course, $S_{0}^{z}$ can become small or zero without its length shrinking as during the first swing in the inverted hierarchy case. It is the length of the polarization vector, not its $z$-component, that is a measure of decoherence. Of course, in the normal hierarchy, $\mathbf{S}_{0}$ performs only small-excursion oscillations so that a significant change of its $z$-component can be achieved only by a change of its length. In this case $\left|\mathbf{S}_{0}\right|$ and $S_{0}^{z}$ are almost identical.

The evolution of $\mathbf{S}_{0}$ was also illustrated in Fig. 1 where we showed its trajectory in the $x$ - $z$-plane. For our chosen geometry where $\mathbf{B}$ is in the $x$ - $z$-plane, $S_{0}^{y}=0$ at all times. For the inverted hierarchy (bottom) we indicate the isotropic-gas trajectory with diamonds at time intervals of $0.01(2 \pi / \kappa)$ where the motion starts in the vertical position. In the half-isotropic case, the particle-number polarization vector $\mathbf{S}_{0}$ spirals in to a position close to the origin. The final offset is very small and depends on the magnitude of $\omega / \mu$ for which we have chosen $10^{-5}$. In 

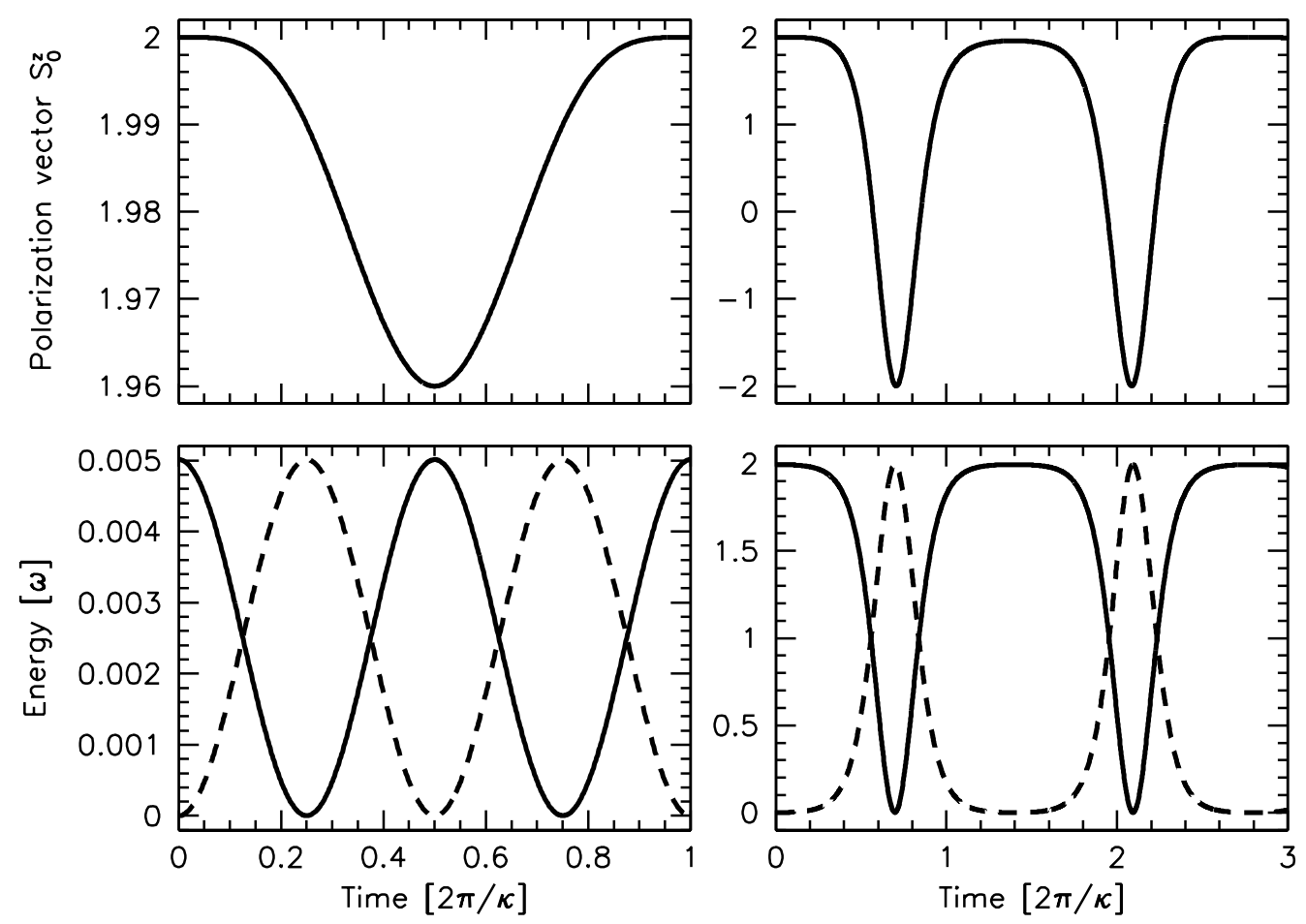

FIG. 2: Evolution of a homogeneous and isotropic ensemble of equal numbers of neutrinos and antineutrinos, initially in the electron flavor, with a vacuum mixing angle $\sin 2 \Theta=0.1$. Top: Polarization vector $S_{0}^{z}$. Bottom: Energy components $E_{\omega}$ (solid) and $E_{\mu}$ (dashed). Left: Normal hierarchy. Right: Inverted hierarchy.
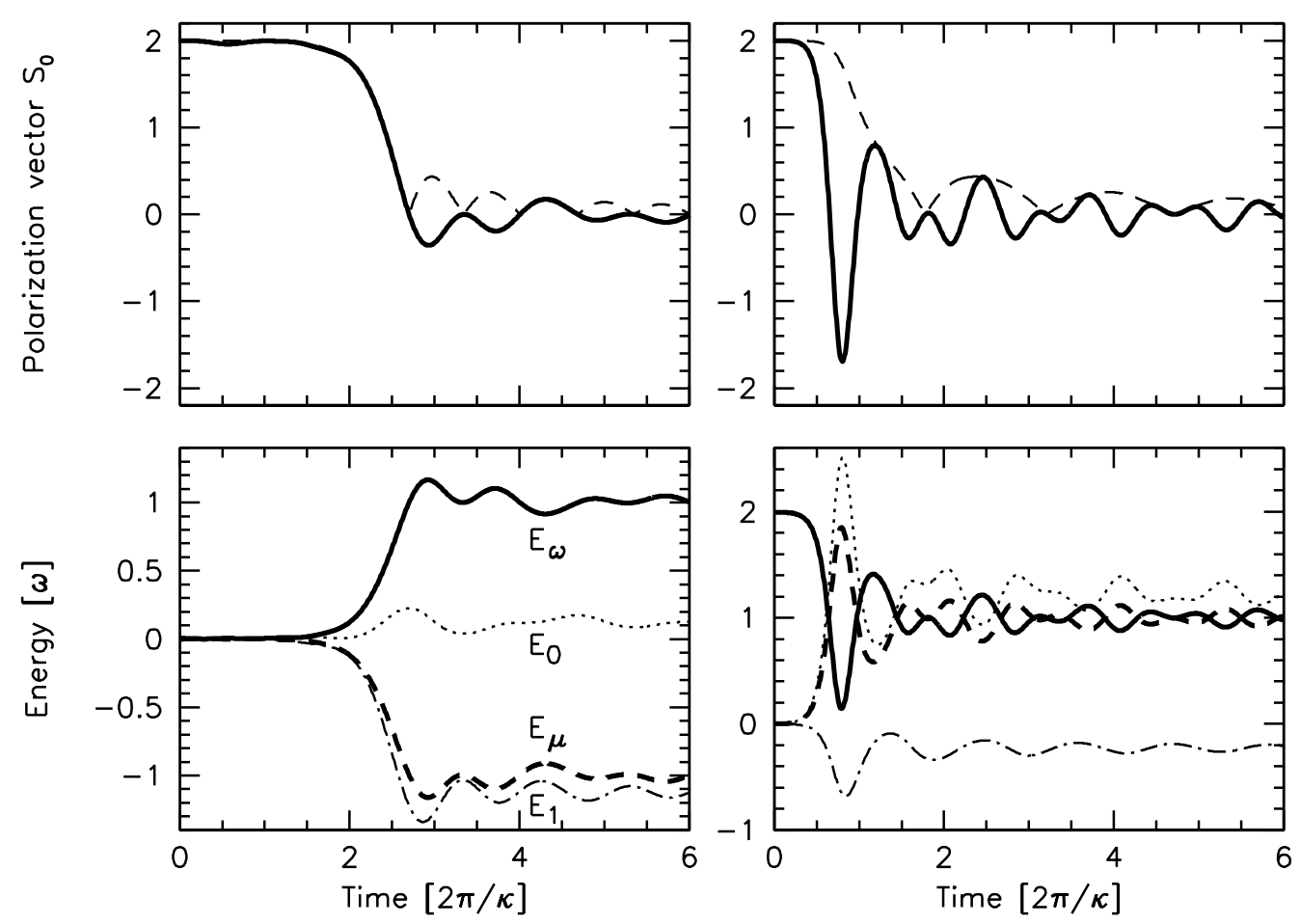

FIG. 3: Same as Fig. 2 now for a half-isotropic neutrino gas. Top: Polarization vector $S_{0}^{z}$ (solid) and the length $\left|\mathbf{S}_{0}\right|$ (dashed). Bottom: Energy components $E_{\omega}$ (solid), $E_{\mu}=E_{0}+E_{1}$ (dashed), $E_{0}$ (thin dotted) and $E_{1}$ (thin dot-dashed). 
the normal hierarchy (top), the isotropic-case motion is a small-excursion harmonic oscillation between the dotted lines. In the half-isotropic case, the evolution begins from the vertical position. A full oscillation back to the vertical position is performed before the shrinking of $\mathbf{S}_{0}$ becomes noticeable.

In the lower panels of Fig. 3 we show the evolution of the different energy components. The simpler case is the inverted hierarchy (right) where the "potential" and "kinetic" energies begin to oscillate as in the isotropic case of Fig. 22 On the time scale of a few bipolar oscillation periods, the two components essentially equipartition, although asymptotically a small offset remains that depends on $\omega / \mu$. Moreover, the neutrino-neutrino energy ("kinetic energy") now develops a nonvanishing flux term $E_{1}=-\mu \mathbf{D}_{1}^{2} / 4$ that inevitably is negative.

The normal hierarchy (left) is initially similar in that $E_{\omega}$ and $E_{\mu}$ oscillate as for a pendulum, even though this motion is not visible on the scale of the plot. As decoherence sets in, a qualitatively different mode of behavior obtains in that the neutrino-neutrino energy $E_{\mu}$ is dominated almost entirely by the negative $E_{1}$ whereas $E_{0}$ now is subdominant. All individual polarization vectors of all modes start aligned with the $z$-direction, i.e., almost aligned with the force direction since the mixing angle is small. Therefore, the initial $E_{\omega}$ is near its minimum. If the overall polarization vector $\mathbf{S}_{0}$ is supposed to shrink, the individual polarization vectors must develop significant deviations from each other and thus the potential energy must increase. Energy conservation then dictates that $E_{\mu}$ becomes negative.

One important conclusion is that the angular dependence of the neutrino-neutrino interaction alone is not enough to cause kinematical decoherence, but its absolute sign is also crucial. If $E_{1}$ were not negative, energy conservation would prevent significant decoherence for the normal hierarchy. Changing this sign in a numerical example indeed reveals the absence of decoherence for the normal hierarchy, but no significant change of behavior for the inverted case. In the real world there is no choice about this sign. It derives from the negative sign of the spatial part in the neutrino current-current interaction, i.e., it is the negative sign inherent in the Lorentz metric. From the equations of motion in the form Eq. (40) one would have never guessed that the absolute sign of the term proportional to $\mathbf{D}_{1}$ plays a crucial role.

\section{Evolution of multipoles}

It is also instructive to study the evolution of the higher multipole components $\mathbf{S}_{n}$. We consider the same example as in the previous section. For a half-isotropic gas the initial relative length of the multipole components is

$$
\begin{aligned}
\frac{S_{1}}{S_{0}} & =\frac{1}{2}, \\
\frac{S_{2 m}}{S_{0}} & =0,
\end{aligned}
$$

$$
\begin{aligned}
\frac{S_{2 m+1}}{S_{0}} & =\frac{1}{2(m+1)} \prod_{k=1}^{m}\left(\frac{1}{2 k}-1\right) \\
& =\frac{(-1)^{m}}{2(m+1)} \frac{\Gamma\left(m+\frac{1}{2}\right)}{\Gamma\left(\frac{1}{2}\right) \Gamma(m+1)},
\end{aligned}
$$

where $S_{n}=\left|\mathbf{S}_{n}\right|$ and all of them are initially oriented in the $z$-direction. Here, $m$ is an integer, i.e., the even multipoles vanish initially except for $S_{0}$.

Instead of using these initial values, we calculate the evolution for a case where all multipoles vanish initially except for $S_{0}=2$ and $S_{1}=1$, i.e., we use the same density and flux terms as in the half-isotropic case. The evolution is very similar. We show the evolution of the first 21 multipoles in Fig. 4 for the inverted hierarchy; the picture is similar for the normal hierarchy. The initial horizontal part of the curves corresponds to their zero initial value so that the offset by 0.1 vertical units between the curves is directly apparent.

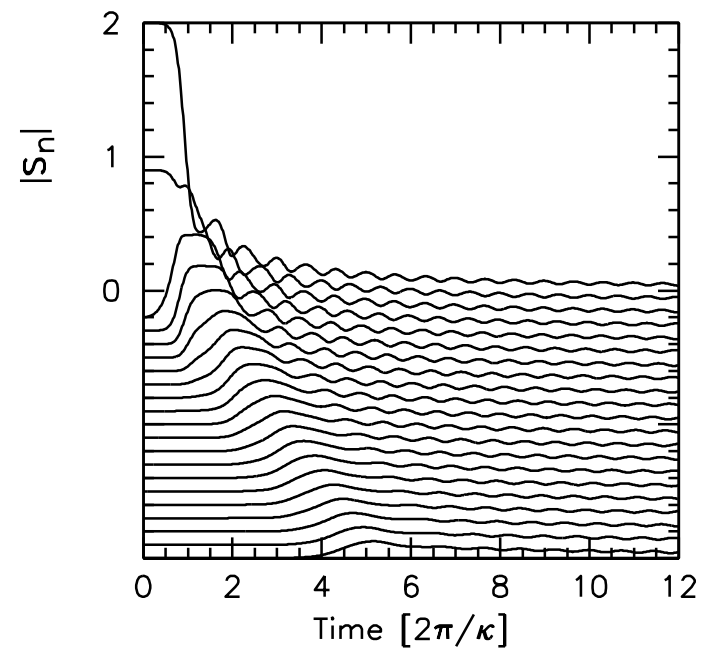

FIG. 4: Evolution of $\left|\mathbf{S}_{n}\right|$ for the first 21 multipoles in the quasi-halfisotropic example with inverted hierarchy. The curves are offset from each other by 0.1 vertical units.

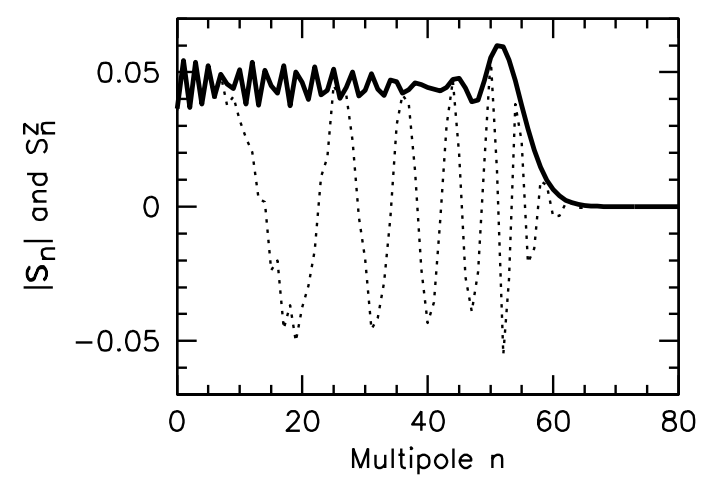

FIG. 5: $\left|\mathbf{S}_{n}\right|$ (solid) and $S_{n}^{z}$ (dotted) as a function of $n$ at $t=12$ for the example of Fig. 4 . 
Initially, $S_{0}$ and $S_{1}$ are large, but quickly decay away by decoherence, whereas the higher multipoles vanish initially and get excited one after another, but then decrease again. The length of each multipole sports "wiggles," i.e., the evolution is not monotonic. Moreover, they have large relative angles in the $x-z$ plane (not shown here), i.e., the spread of the inital excitation to larger and larger multipoles is far from simple in detail, but the overall process is as expected. In Fig. [5 we show $\left|\mathbf{S}_{n}\right|$ and $S_{n}^{z}$ as a function of $n$ at $t=12$ for the example of Fig. 4. At $t=12$, multipoles $n \gtrsim 60$ are not yet excited. The nearly linear increase of the phases between individual polarization vectors corresponds here to a diffusion of the "multipole wave" to larger $n$ with approximately constant speed, $n \simeq \kappa t$.

Qualitatively, this can be understood as follows. The second line in Eq. (40) together with the analogue of Eq. (24) implies that

$$
\begin{aligned}
\dot{\mathbf{D}}_{n} \simeq \omega \mathbf{B} \times \mathbf{S}_{n} & -\mu \mathbf{D}_{1} \times\left(\frac{\mathbf{D}_{n}^{\prime}}{2 n+1}+\frac{\mathbf{D}_{n}^{\prime \prime}}{2}\right) \\
& +\mu\left(\mathbf{D}_{0}-\mathbf{D}_{1}\right) \times \mathbf{D}_{n} .
\end{aligned}
$$

The last term does not contribute to the growth of $\left|\mathbf{D}_{n}\right|$ and can be ignored. Approximate equipartition between potential and kinetic energies in Eq. (44) implies $\left|\mathbf{D}_{0}\right| \sim\left|\mathbf{D}_{1}\right| \sim 2(\omega / \mu)^{1 / 2}$, as indeed was observed in Fig. 3. The second term in Eq. (50) then represents a combination of a drift in $n$-space with velocity $\sim \kappa /(\sqrt{2} n)$ and diffusion with diffusion coefficient $\sim \kappa / \sqrt{2}$. Up to a factor $t^{-1 / 2}$ this gives rise to an exponential factor $\sim \exp \left[-n^{2} /(2 \sqrt{2} \kappa t)\right]$. On the other hand, since $\left|\mathbf{S}_{n}\right| \sim(\mu / \omega)^{1 / 2}\left|\mathbf{D}_{n}\right|$ for $n \geq 2$, the first term in Eq. (50) can give rise to exponential growth whenever $\left(\mathbf{B} \times \mathbf{S}_{n}\right) \cdot \mathbf{D}_{n}>0$. This growth will be non-monotonic, but can roughly be estimated as $\propto \exp (\kappa t / 2)$ before $\mathbf{D}_{n}$ saturates. Using perturbation theory, we will find a similar growth rate for $\mathbf{D}_{1}$ at early times in Sect. IVB Combining these two factors, one sees that at time $t$ the wave front of $\mathbf{D}_{n}$, and thus also of $\mathbf{S}_{n}$, should be located at $n \simeq \kappa t$, which, according to Figs. 4 and 5 indeed it is within about $20 \%$. The slow decrease of $\mathbf{S}_{n}$ and $\mathbf{D}_{n}$ at late times can be interpreted as due to the $t^{-1 / 2}$ factor in the diffusive behavior.

\section{A simple model of decoherence}

The overall behavior of the inverted-hierarchy case is qualitatively easy to understand. To this end we consider an ensemble of polarization vectors $\mathbf{P}_{u}$, initially oriented in the $z$-direction, that rotate around the $y$-axis, each with a frequency $u$, so that

$$
\begin{aligned}
& P_{u}^{z}=\cos (u t), \\
& P_{u}^{x}=\sin (u t) .
\end{aligned}
$$

Both $u$ and $t$ are normalized to be dimensionless.

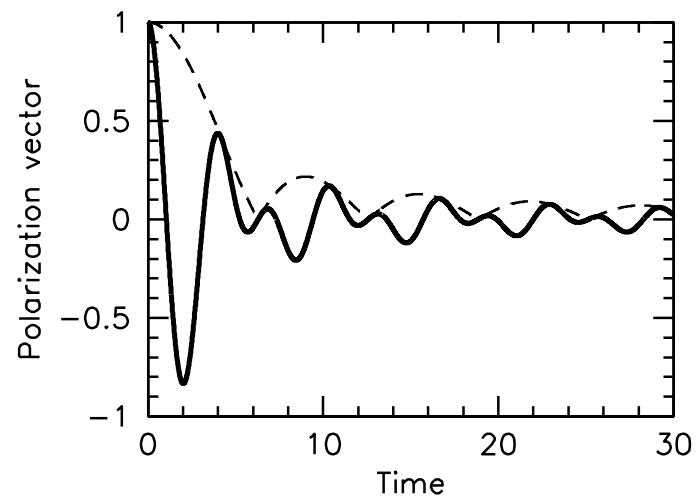

FIG. 6: Evolution of $P_{0}^{z}$ (solid) and $\left|\mathbf{P}_{0}\right|$ (dashed) of our toy model with $\Delta u=1$.

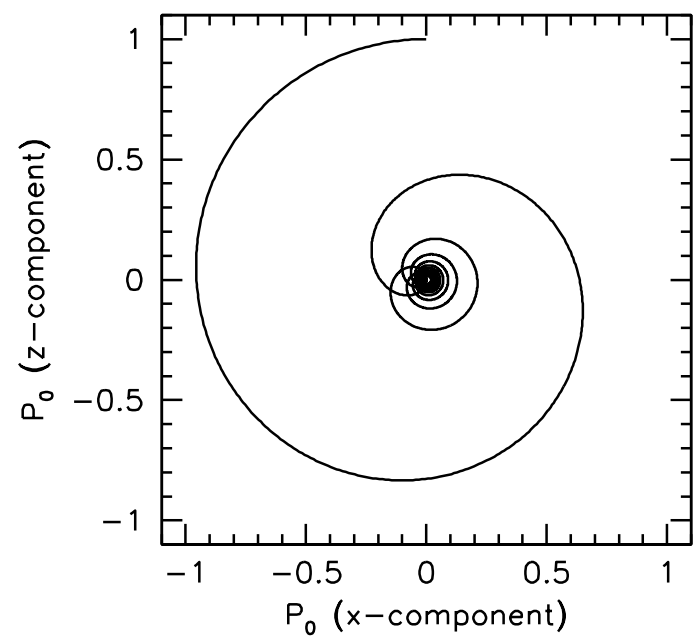

FIG. 7: Trajectory of $\mathbf{P}_{0}$ in the $x$ - $z$-plane for our toy model of decoherence with $\Delta u=1$.

If the frequencies are spread over $1 \leq u \leq 1+\Delta u$, then the overall polarization vector evolves as

$$
\begin{aligned}
P_{0}^{z} & =\int_{1}^{1+\Delta u} d u \cos (u t)=\frac{\sin [(1+\Delta u) t]-\sin (t)}{t} \\
P_{0}^{x} & =\int_{1}^{1+\Delta u} d u \sin (u t)=\frac{\cos (t)-\cos [(1+\Delta u) t]}{t} \\
\left|\mathbf{P}_{0}\right| & =\left[\left(P_{0}^{x}\right)^{2}+\left(P_{0}^{z}\right)^{2}\right]^{1 / 2}=\frac{2|\sin (\Delta u t / 2)|}{t}
\end{aligned}
$$

For $\Delta u=1$ we show in Fig. 6 the evolution of $P_{0}^{z}$ (solid line) and the length $\left|\mathbf{P}_{0}\right|$ (dashed line). In Fig. 7 we show the trajectory of $\mathbf{P}_{0}$ in the $x$ - $z$-plane.

Unsurprisingly, this simple model represents qualitatively the features of the inverted-hierarchy case. In particular, the trajectory in the $x$ - $z$-plane is not a true spiral, but there are many crossings of the origin as the length of the overall polarization vector shrinks. Its length does not shrink monotonically. Its envelope decreases as a power law $t^{-1}$, not exponentially. 
This example also shows that it is not trivial to define a useful measure of kinematical decoherence. The $z$-component of the polarization vector is not useful because $\mathbf{P}_{0}$ can tilt so that its $z$-component vanishes, i.e., both flavors are present with the same probability, yet the flavor content of the ensemble is perfectly coherent. The length of $\mathbf{P}_{0}$ is a much better measure in analogy to dynamical decoherence of a single mode where the length of $\mathbf{P}_{\mathbf{p}}$ is a measure of dynamical decoherence; unit length would correspond to a pure state. In our case, the length of the individual $\mathbf{P}_{\mathbf{p}}$ is conserved, whereas the length of the total $\mathbf{P}_{0}$ shrinks, but not monotonically.

It is not evident if there is an "entropy" measure that evolves monotonically as a consequence of kinematical decoherence. We note, however, that the origin of kinematical decoherence is the differential evolution between neighboring polarization vectors. In our toy example, the angle between neighboring polarization vectors grows linearly as $\Delta u t$ and thus is a measure of decoherence.

Motivated by this observation we can define the "winding number"

$$
N=\frac{1}{2 \pi} \int_{-1}^{+1} d u \frac{\left|d \mathbf{P}_{u} / d u\right|}{\left|\mathbf{P}_{u}\right|}
$$

In the symmetric case where the evolution of the polarization vectors is essentially restricted to the $x$-z-plane, this quantity tells us the number of windings around the $y$-direction of the full ensemble of polarization vectors. In Fig. 8 we show the evolution of this quantity for the normal and inverted hierarchy examples of Fig. 3, $N$ counts how often the length of $\mathbf{S}_{0}$ shrinks to zero.

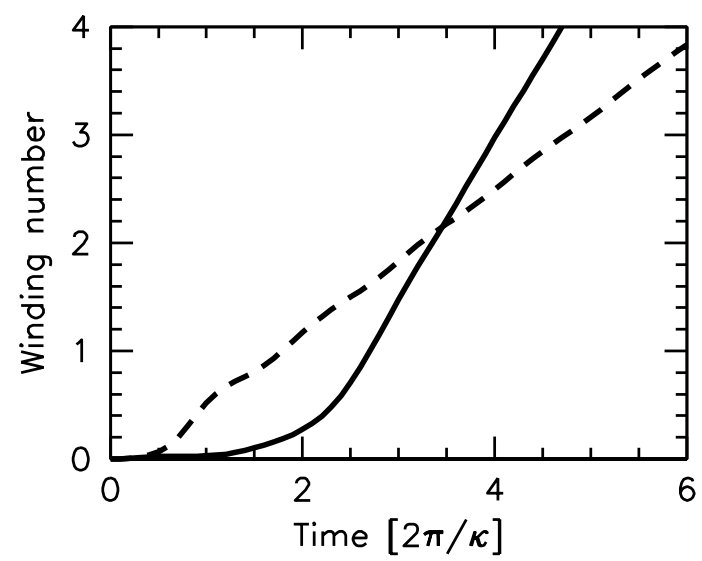

FIG. 8: Evolution of the "winding number" as defined in Eq. (53) for the half-isotropic case of Fig. 3. Normal hierarchy (solid) and inverted hierarchy (dashed).

\section{E. Recurrence effects}

Closely related to kinematical decoherence are recurrence effects that arise when one uses a limited number of polarization vectors, i.e., when the full ensemble is represented by a coarse-grained (binned) ensemble of polarization vectors or, in multipole space, when the series is truncated at some multipole $n_{\max }$. Binning or truncation are unavoidable in numerical treatments and represent equivalent approximations. ${ }^{2}$

If the phase differences evolve linearly between different polarization vectors as in the toy example of the previous section, then the initial state will recur when each polarization vector has turned around the $y$-axis by $2 \pi$ relative to its neighbors. Put another way, if we use $n_{\text {pol }}$ bins to represent the polarization vectors, we expect recurrence to begin when $N=\left(n_{\text {pol }}-1\right) / 2$. We demonstrate this effect in Fig. 9 for the same example as in Fig. 3, normal hierarchy, using $n_{\text {pol }}=51$. Indeed, the winding number increases until approximately $N=25$ and then decreases back to almost zero, and so forth. This system is almost periodic on the recurrence time scale, reflecting that nonlinear effects play a subleading role here. Since a large flux term is present from the start,
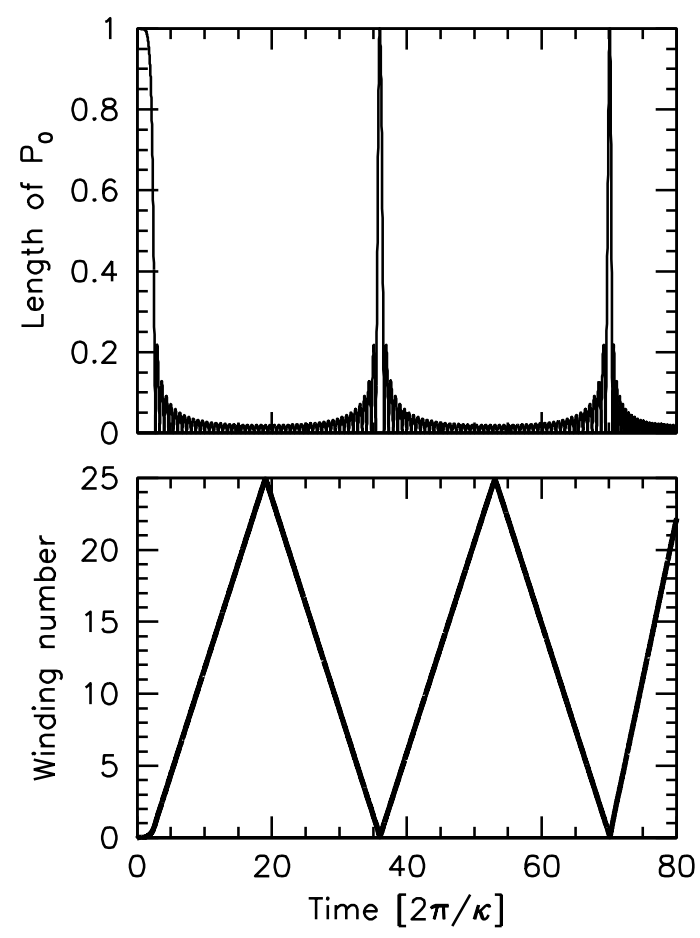

FIG. 9: Evolution of $\left|\mathbf{P}_{0}\right|$ and the winding number for the normal-hierarchy case of Fig. 3 with 51 polarization vectors.

\footnotetext{
2 In Ref. 11] a multipole expansion ("partial wave expansion") was proposed and dismissed with the argument that the necessary truncation would introduce uncontrolled numerical effects whereas coarse bins in angle space would provide limited resolution, yet a correct solution of the nonlinear equations of motion. We disagree with this assessment. The same unphysical recurrence effects occur both if the number of angular bins or the multipole order of truncation is too small.
} 
kinematical decoherence is very similar to the linear toy example of the previous section where recurrence would be exact and the system would be periodic with a frequency proportional to $\left(n_{\text {pol }}-1\right)^{-1}$ times the individual polarization vector's oscillation frequency.

Using the multipoles as the primary variables, the same recurrence effects occur due to the truncation $n_{\max }$ of the multipole series. The "multipole wave" of Fig. 5 can only propagate up to $n_{\max }$, is then reflected, and eventually returns back to $n=0$. This is the moment of maximum recurrence of the overall polarization vector.

For a more complicated example such as a realistic simulation of the supernova case, recurrence effects may not be so obvious as here. Therefore, in a numerical treatment one must make sure that the largest relative angle developed between any two neighboring polarization vectors never grows to order unity, i.e., one should monitor the largest relative angle between neighboring polarization vectors as a measure of accuracy of the calculation. In other words, kinematical decoherence is a property of the entire ensemble, but can also be a a property of individual bins if they are too coarse. One must make sure that within all individual bins kinematical decoherence remains negligible.

\section{SMALL INITIAL ANISOTROPY}

\section{A. Numerical example}

We next turn to a symmetric system that is perfectly isotropic except for an arbitrarily small but nonzero initial anisotropy. We work in the multipole picture and assume that initially $\mathbf{D}_{n}=0$ for all $n, \mathbf{S}_{n}=0$ for $n \geq 2$, $S_{0}^{z}=2$ and $S_{1}^{z}=\xi \ll 1$. Note that $\xi=1$ corresponds to a flux term equivalent to the half-isotropic case.

As a first example we show in Fig. 10 the evolution of the overall polarization vector $\mathbf{S}_{0}$ (top) for the normal hierarchy (left) and the inverted hierarchy (right). The initial anisotropy is $\xi=10^{-4}$ and $\sin 2 \Theta=0.1$. For both hierarchies, kinematical decoherence eventually obtains.

In the bottom panels we show the evolution of $-E_{1}=$ $(\mu / 4) \mathbf{D}_{1}^{2}$ (solid line) as a proxy for $\mathbf{D}_{1}$ which is the primary quantity that causes decoherence. For the symmetric neutrino gas only the component $D_{1}^{y}$ is nonzero, but it can be negative and can change sign so that it is difficult to display on a logarithmic scale. We also show the evolution of the "potential energy" $E_{\omega}$ (dotted line).

For the normal hierarchy, $D_{1}^{y}$ grows exponentially after a short initial transient. While $D_{1}^{y}$ is small, the potential energy $E_{\omega}$ performs the usual oscillations of a harmonic oscillator, complemented by the opposite oscillations of the kinetic energy $E_{\mu}=E_{0}-E_{1}$ (not shown) that is entirely dominated by $E_{0}$. As $D_{1}^{y}$ grows, it eventually dominates the kinetic energy and the end state is approximately $E_{\omega}=+\omega$ and $E_{\mu}=-\omega$, with $E_{1}$ dominating $E_{\mu}$. In other words, the end state is exactly as in the previous case with a large initial anisotropy. The crucial novel feature is that the flux term grows exponentially from a small value. This is a purely nonlinear effect caused by neutrino-neutrino interactions. For smaller mixing angles and/or other values of $\xi$, the behavior is analogous. The rising part of the $E_{1}$ curve is the same, except that it shifts vertically in direct proportion to $\xi$ and $\sin 2 \Theta$.

For the inverted hierarchy, the evolution is far more complicated. While $D_{1}^{y}$ grows in an average sense, this evolution is overlaid with oscillations and in fact $D_{1}^{y}$ changes sign at each spike in the $E_{1}$ curve. Moreover, the evolution of the envelope of the $E_{1}$ curve is not monotonic. It can perform complicated motions, with growing, declining, and nearly flat phases, until finally decoherence obtains.

From these numerical observations we tentatively conclude that an isotropic neutrino ensemble of equal densities of neutrinos and antineutrinos is not stable. A small flux term triggers a run-away evolution towards kinematical decoherence.

\section{B. Analytic treatment}

\section{Simplified equations of motion}

As a starting point for an analytic understanding of this instability we use the equations of motion Eq. (40) for the vectors $\mathbf{S}_{n}$ and $\mathbf{D}_{n}$. Initially only $\mathbf{S}_{0}$ is of order one, $\left|\mathbf{S}_{1}\right|=\xi \ll 1$, and all others vanish. As long as $\mathbf{D}_{1}$ is sufficiently small, none of the higher multipoles can grow large so that we can limit our attention to the equations for $n=0$ and $n=1$, i.e., to Eqs. (41) and (42).

We further observe that the $n=0$ equations are coupled to the higher multipoles only by the term $-\mu \mathbf{D}_{1} \times \mathbf{S}_{1}$, consisting of a product of two small quantities, i.e., it is at least of order $\xi^{2}$. Therefore, the evolution of the nearisotropic ensemble is identical with that of the isotropic case until $\mathbf{D}_{1}$ has grown sufficiently large. In other words, up to second order in $\xi$ the $n=0$ equation is that of the isotropic case.

Therefore, what remains to be solved is the $n=1$ equation. After neglecting terms involving $n \geq 2$ all we need to study is

$$
\begin{aligned}
& \dot{\mathbf{S}}_{1}=\left(\omega \mathbf{B}+\frac{\mu}{3} \mathbf{S}_{0}\right) \times \mathbf{D}_{1}+\mu \mathbf{D}_{0} \times \mathbf{S}_{1} \\
& \dot{\mathbf{D}}_{1}=\omega \mathbf{B} \times \mathbf{S}_{1}+\frac{2 \mu}{3} \mathbf{D}_{0} \times \mathbf{D}_{1}
\end{aligned}
$$

where $\mathbf{S}_{0}(t)$ and $\mathbf{D}_{0}(t)$ are the solutions of the unperturbed pendulum equations.

The equations simplify further in our case of equal $\nu$ and $\bar{\nu}$ densities where symmetry dictates that all polarization vectors $\mathbf{P}_{\mathbf{p}}$ and $\overline{\mathbf{P}}_{\mathbf{p}}$ evolve as each other's mirror images relative to the $x$ - $z$-plane, the latter being singled out by our choice that $\mathbf{B}$ lies in that plane. In this case all $\mathbf{D}_{n}$ vectors are parallel to the $y$-axis, whereas all $\mathbf{S}_{n}$ vectors are confined to the $x$ - $z$-plane. 

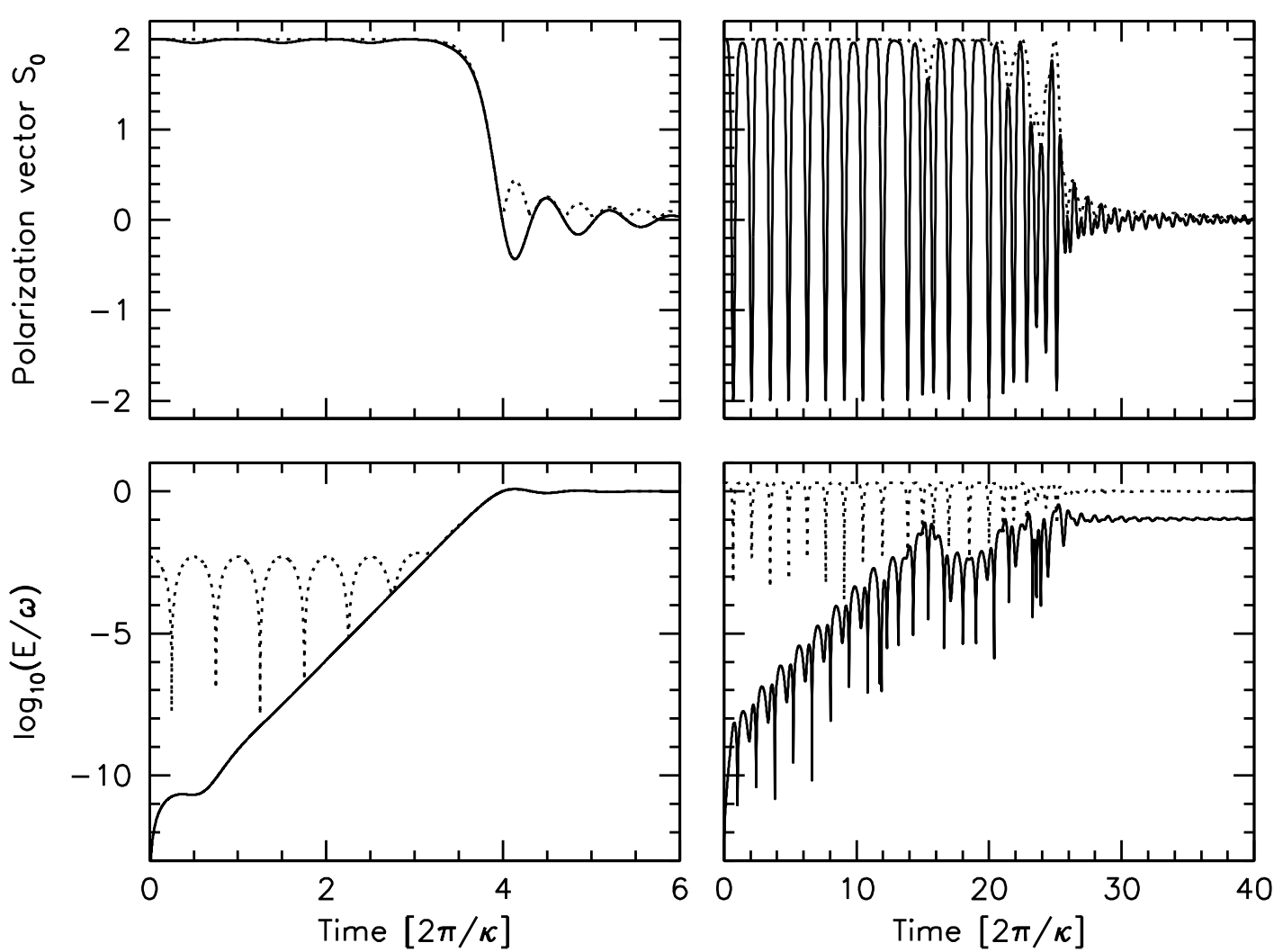

FIG. 10: Evolution of a homogeneous and near-isotropic ensemble of equal $\nu$ and $\bar{\nu}$ densities with $\sin 2 \Theta=0.1$ as in Figs. 2 and 3 . The initial anisotropy is $\xi=10^{-4}$. Top: Polarization vector $S_{0}^{z}$ (solid) and the length $\left|\mathbf{S}_{0}\right|$ (dotted). Bottom: Neutrino-neutrino flux energy $-E_{1}$ (solid) and potential energy $E_{\omega}$ (dotted). Left: Normal hierarchy. Right: Inverted hierarchy.

As a consequence, the $\mathbf{D}_{0} \times \mathbf{D}_{1}$ term drops out. The second equation then implies $\ddot{\mathbf{D}}_{1}=\omega \mathbf{B} \times \dot{\mathbf{S}}_{1}$, and with the first equation yields

$$
\ddot{\mathbf{D}}_{1}=\omega \mathbf{B} \times\left[\left(\omega \mathbf{B}+\frac{\mu}{3} \mathbf{S}_{0}\right) \times \mathbf{D}_{1}\right]+\omega \mu \mathbf{B} \times\left(\mathbf{D}_{0} \times \mathbf{S}_{1}\right) .
$$

Expanding the triple product, observing that in our case $\mathbf{B} \cdot \mathbf{D}_{n}=0$, and using $\mathbf{B}^{2}=1$ this is

$$
\ddot{\mathbf{D}}_{1}=-\left(\omega^{2}+\frac{\omega \mu}{3} \mathbf{B} \cdot \mathbf{S}_{0}\right) \mathbf{D}_{1}+\omega \mu\left(\mathbf{B} \cdot \mathbf{S}_{1}\right) \mathbf{D}_{0} .
$$

We repeat that all $\mathbf{D}_{n}$ are here parallel to each other and to the $y$-axis. In the absence of neutrino-neutrino interactions $(\mu=0), \mathbf{D}_{1}$ obeys a harmonic-oscillator equation.

In a dense neutrino gas, $\mu \gg \omega$, we may ignore the term proportional to $\omega^{2}$. Since during the growth phase, $\left|\mathbf{D}_{1}\right| \lesssim\left|\mathbf{D}_{0}\right|$ and $\left|\mathbf{S}_{1}\right| \ll\left|\mathbf{S}_{0}\right|$, the last term in Eq. (56) can also be neglected. A possible exponential growth is then accounted for by the term

$$
\ddot{\mathbf{D}}_{1}=-\frac{\kappa^{2}}{3} \frac{\mathbf{B} \cdot \mathbf{S}_{0}}{2} \mathbf{D}_{1},
$$

where we have used the bipolar oscillation frequency $\kappa^{2}=$ $2 \omega \mu$ and we note that initially $\left|\mathbf{S}_{0}\right|=2$ and that its length remains nearly constant if $|\sin 2 \Theta| \ll 1$ unless there is kinematical decoherence.

\section{Normal hierarchy}

If the mixing angle is small, the "normal hierarchy" is defined by neutrinos being essentially in the lower mass eigenstate or $\mathbf{B} \cdot \mathbf{S}_{0} / 2=-1$ up to corrections of order $\sin ^{2} 2 \Theta$. Therefore, the critical part of the equation of motion for the flux term is approximately

$$
\ddot{\mathbf{D}}_{1}=+\frac{\kappa^{2}}{3} \mathbf{D}_{1} .
$$

The solution includes growing modes of the form

$$
\mathbf{D}_{1} \propto \exp \left(+\frac{2 \pi}{\sqrt{3}} \tau\right)
$$

where $\tau=\kappa t / 2 \pi$ is the dimensionless time variable used in all of our plots, i.e., time in units of the bipolar oscillation period. This behavior is numerically verified in the lower-left panel of Fig. 10 if we recall that the plotted quantity is $-E_{1} \propto \mathbf{D}_{1}^{2} \propto \exp [(4 \pi / \sqrt{3}) \tau]$.

Once more we note that the crucial absolute sign in Eq. (58) is traced back to the negative sign in the term $\left(1-\mathbf{v}_{\mathbf{q}} \cdot \mathbf{v}_{\mathbf{p}}\right)$ in Eq. (5). Therefore, the instability of the near-isotropic neutrino gas, for the normal hierarchy, is not just caused by different angular modes experiencing 
different neutrino-neutrino effects. This would also be the case for a hypothetical term $\left(1+\mathbf{v}_{\mathbf{q}} \cdot \mathbf{v}_{\mathbf{p}}\right)$ that naively looks very similar, but causes a completely different overall behavior. In more complicated numerical situations, e.g., for a full supernova simulation, it is easy to change this sign in order to diagnose the relevance of the term Eq. (57) for the overall behavior of the system.

Initially $\mathbf{D}_{1}=0$ so that the exponential growth term alone is not enough. The initial evolution is dominated by the second line of Eq. (54) that is initially

$$
\dot{D}_{1}=\left(\frac{\omega}{\mu}\right)^{1 / 2} \frac{\kappa}{\sqrt{2}} \xi \sin 2 \Theta
$$

where $D_{1}=\left|\mathbf{D}_{1}\right|$ and we have used $|\mathbf{B}|=1$ and $\mathbf{S}_{1}(0)=$ $(0,0, \xi)$. Here the absolute sign is not critical because it does not matter if $\mathbf{D}_{1}$ grows in the positive or negative $y$ direction. The first factor $(\omega / \mu)^{1 / 2}$ sets the overall scale for $\mathbf{D}_{1}$. From the expression for the energy we note that $\mathbf{S}$ and $\mathbf{D}$ appear in the combinations $\omega\left|\mathbf{S}_{0}\right|$ and $\mu \mathbf{D}_{0}^{2}$ or $\mu \mathbf{D}_{1}^{2}$. The quantities $\mathbf{S}_{n}$ are of order unity, whereas the natural scale for $\mathbf{D}_{n}$ is $(\omega / \mu)^{1 / 2}$. The initial evolution is

$$
\left(\frac{\mu}{\omega}\right)^{1 / 2} \frac{D_{1}}{\xi \sin 2 \Theta}=\frac{2 \pi}{\sqrt{2}} \tau,
$$

where, again, $\tau=(\kappa / 2 \pi) t$ is our usual measure of time. This linear growth is shown in Fig. 11] as a dotted line and agrees with the numerical examples.

Besides a transient caused by the other terms in the equation, the exponential growth takes over after a time of order $2 \pi / \kappa$. We conclude that the initial value of $(\mu / \omega)^{1 / 2} D_{1}$ for its exponential growth is of order $\xi \sin (2 \Theta)$ so that it grows to order unity within a time scale of order $-(2 \pi / \kappa) \ln (\xi \sin 2 \Theta)$.

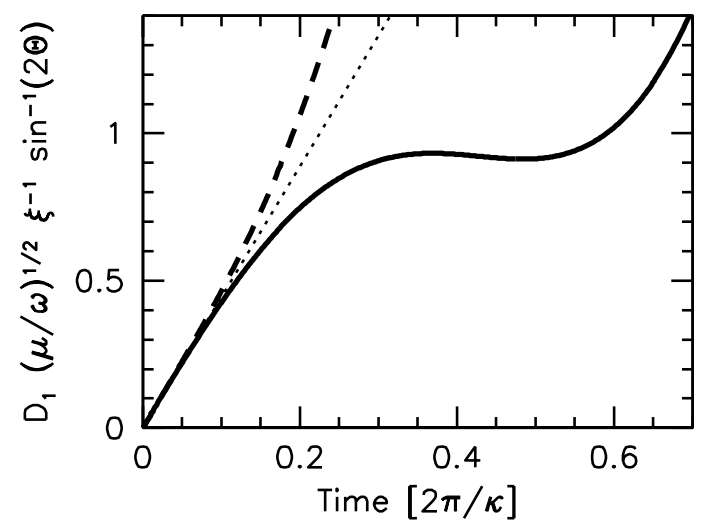

FIG. 11: Initial evolution of $\mathbf{D}_{1}$ as discussed in the text for the normal hierarchy (solid) and the inverted hierarchy (dashed). The dotted line is the linear relationship of Eq. (61).

\section{Inverted hierarchy}

For the inverted hierarchy, the initial growth of $D_{1}$ is the same (dashed line in Fig. 11). After that, however, the situation is entirely different because initially $\mathbf{B} \cdot \mathbf{S}_{0} / 2=+1$. This corresponds to the pendulum starting in a nearly upright position from where it starts almost full-circle oscillations. For our usual assumption $|\sin 2 \Theta| \ll 1$ the term $\mathbf{B} \cdot \mathbf{S}_{0}$ is almost identical with $S_{0}^{z}$ shown in the upper-right panel of Fig. 10, This term changes sign during every swing of the pendulum. During the phases when $\mathbf{B} \cdot \mathbf{S}_{0}>0, \mathbf{D}_{1}$ oscillates whereas during the phases $\mathbf{B} \cdot \mathbf{S}_{0}<0$ exponential growth obtains. These are the relatively short phases when the pendulum is oriented downward, whereas it stays upright for long periods if the mixing angle is small.

We illustrate this behavior in Fig. 12 where we show the evolution of $D_{1}$ overlaid with that of $S_{0}^{z}$. We have chosen an extremely small mixing angle, $\sin 2 \Theta=10^{-7}$, to obtain long plateaus for $S_{0}^{z}$ where the pendulum stands almost still in an almost upright position. During these phases we have $\mathbf{B} \cdot \mathbf{S}_{\mathbf{0}} / 2=+1$ so that Eq. (57) implies harmonic oscillations of $D_{1}$ with the frequency $\kappa / \sqrt{3}$ as borne out by the example in Fig. 12,

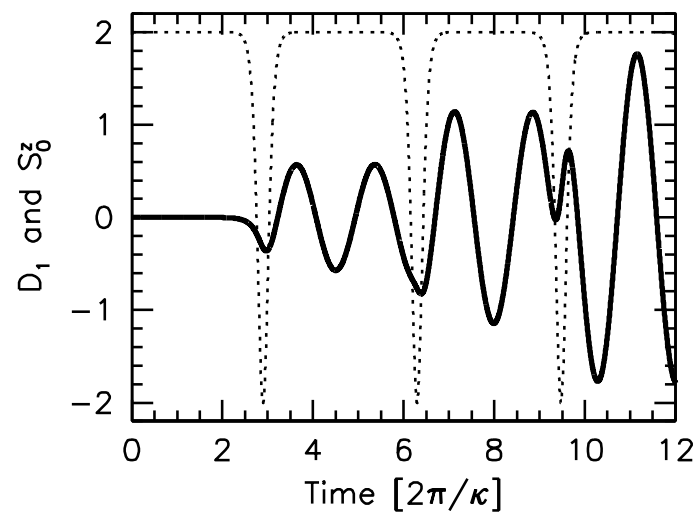

FIG. 12: Initial evolution of $\mathbf{D}_{1}$ (solid line, arbitrary units) and $S_{0}^{z}$ (dotted) for the inverted hierarchy with $\xi=10^{-4}$ and $\sin 2 \Theta=10^{-7}$.

The frequency $\kappa / \sqrt{3}$ is not matched to the pendulum's oscillation period because the duration of the "plateaus" depend logarithmically on $\sin 2 \Theta$. Therefore, the short exponential growth phases when $S_{0}^{z}<0$ occur at erratic instances relative to the harmonic $\mathbf{D}_{1}$ oscillation. This interplay explains the erratic behavior of the $\mathbf{D}_{1}$ evolution that is apparent in Fig. 10 and that obtains in all numerical examples. This interplay also explains why small changes of parameters such as $\sin 2 \Theta$ can completely change the overall $\mathbf{D}_{1}$ evolution.

Therefore, we cannot prove if there is some specific combination of parameters where kinematical decoherence will not occur, although this would have to be isolated parameter points that presumably have measure zero in parameter space if they exist at all. 
Except for this caveat we conclude that both for the normal and inverted hierarchy kinematical decoherence is an unavoidable consequence of the nonlinear neutrinoneutrino interaction terms. An infinitesimally small, but nonzero, deviation from isotropy is enough to trigger an exponential evolution towards flavor equilibrium. Therefore, the pendulum solution that describes the behavior of a perfectly isotropic gas is merely an unstable limit cycle of this nonlinear system.

\section{CONCLUSIONS AND OUTLOOK}

We have investigated multi-angle kinematical decoherence effects in a dense neutrino gas consisting of equal $\nu$ and $\bar{\nu}$ densities. The current-current nature of the weakinteraction Hamiltonian implies that a current of the background medium causes kinematical decoherence between neutrinos propagating in different directions. This simple effect becomes entirely nontrivial in the most interesting case when the "background current" is caused by the neutrinos themselves.

We have shown that a neutrino gas of this sort is not stable in flavor space. If one prepares the ensemble in a given flavor state, even a small deviation from isotropy is enough to trigger an exponential evolution towards flavor equipartition, even when the mixing angle is small. Up to logarithmic corrections, the time scale is determined by the bipolar oscillation period. We thus have to do with a self-induced macroscopic pair conversion process that proceeds much faster than ordinary pair processes that occur at order $G_{\mathrm{F}}^{2}$.

With hindsight this is probably the same "speed-up effect" discussed in Ref. [11]. However, the fast speed $\kappa=\sqrt{2 \omega \mu}$ is not introduced by the multi-angle effect as is perhaps suggested in Ref. [11]. Rather, $\kappa$ is the bipolar oscillation frequency that is inherent even in the isotropic system. For the normal hierarchy "nothing" seems to happen in the isotropic case, although this would be a misperception because the system moves with the speed $\kappa$, but the amplitude is small if $\sin 2 \Theta \ll 1$. In any event, we agree with the importance of multi-angle effects and with the end result conjectured in Ref. [11] that complete flavor equipartition obtains on a time scale $\kappa^{-1}$. However, we have found no indication that an even faster time scale $\mu^{-1}$ plays any role, in contrast to what was conjectured in Ref. [11].

Of course, flavor equipartition obtains only in a macroscopic sense with some degree of coarse graining in phase space. It is the nature of kinematical decoherence that information is not lost and the entropy does not increase. On the other hand, kinematical and dynamical decoherence are not always operationally distinguishable. It is not necessarily possible to distinguish between a neutrino gas in true chemical equilibrium and one where neighboring modes are simply "de-phased" relative to each other.

Either way, the "self-maintained coherence" of a dense neutrino gas represents only an unstable limiting form of behavior of the perfectly isotropic case. Since no gas can be perfectly isotropic, self-induced decoherence is the generic behavior of a symmetric $\nu \bar{\nu}$ gas.

We have only analyzed the two-flavor case. It remains to be studied how our results carry over to a genuine three-flavor situation.

Contrary to a naive expectation, the fact that neutrinos propagating in different directions experience different weak potentials is necessary, but not sufficient, to cause multi-angle decoherence. The negative sign in the Lorentz metric plays a crucial role because it determines that the flux term grows exponentially rather than oscillating. The occurrence of kinematical decoherence is a subtle feature of the nonlinear equations.

We have studied the simplest possible case where decoherence effects obtain. A more realistic case is provided by neutrinos streaming off a supernova core. In this case the fluxes of neutrinos and antineutrinos are different and geometry implies that the neutrino-neutrino interaction strength declines with the fourth power of radius. The evolution of an asymmetric system is far more complicated, even in the isotropic case, where the simple pendular motion turns into one of a spinning top that can precess, nutate, or simply swing, depending on the $\nu \bar{\nu}$ asymmetry. In the supernova context, transitions between these modes occur as $\mu$ declines as a function of radius. Multi-angle decoherence cannot occur, for example, in regions where synchronized oscillations prevail.

Numerical simulations suggest that for neutrinos streaming off a supernova core, the collective behavior characteristic of an isotropic gas prevails in many situations, i.e., kinematical decoherence occurs, but is not the dominating feature of the system, contrary to what we have found here for the symmetric $\nu \bar{\nu}$ gas. Evidently it is not straightforward to apply the insights gained from our present study to the supernova case.

We imagine, however, that the methods developed here may take us one step further to understand the supernova problem. We have formulated the equations of motion in a novel form adapted to the problem of multi-angle propagation, i.e., we have used a multipole expansion rather than the usual momentum modes and we have used the particle number and lepton number polarization vectors (or density matrices) as the fundamental variables. In this way, the crucial exponentially growing quantity, our $\mathbf{D}_{1}$, could be isolated as the primary cause of kinematical decoherence. This approach may prove useful for understanding the supernova problem as well.

For numerical three-flavor studies we have provided an expression for the conserved energy of the system in terms of the density matrices. The conserved energy and its three components $E_{\omega}, E_{0}$ and $E_{1}$ provide a useful diagnostic tool for the behavior of the system.

Even simple nonlinear systems can exhibit a surprisingly rich phenomenology. It is fascinating that the neutrinos streaming off a supernova core provide an intriguing case in point where many aspects of its behavior remain to be understood. 


\section{Acknowledgments}

This work was partly supported by the Deutsche Forschungsgemeinschaft under Grants No. SFB-375 and
TR-27 and by the European Union under the ILIAS project, contract No. RII3-CT-2004-506222.
[1] J. Pantaleone, "Neutrino oscillations at high densities," Phys. Lett. B 287, 128 (1992).

[2] S. Samuel, "Neutrino oscillations in dense neutrino gases," Phys. Rev. D 48, 1462 (1993).

[3] V. A. Kostelecký, J. Pantaleone and S. Samuel, "Neutrino oscillation in the early universe," Phys. Lett. B 315, 46 (1993).

[4] V. A. Kostelecký and S. Samuel, "Neutrino oscillations in the early universe with an inverted neutrino mass hierarchy," Phys. Lett. B 318, 127 (1993).

[5] V. A. Kostelecký and S. Samuel, "Nonlinear neutrino oscillations in the expanding universe," Phys. Rev. D 49, 1740 (1994).

[6] V. A. Kostelecký and S. Samuel, "Self-maintained coherent oscillations in dense neutrino gases," Phys. Rev. D 52, 621 (1995) hep-ph/9506262.

[7] V. A. Kostelecký and S. Samuel, "Neutrino oscillations in the early universe with nonequilibrium neutrino distributions," Phys. Rev. D 52, 3184 (1995) hep-ph/9507427.

[8] S. Samuel, "Bimodal coherence in dense selfinteracting neutrino gases," Phys. Rev. D 53, 5382 (1996) hep-ph/9604341.

[9] V. A. Kostelecký and S. Samuel, "Nonequilibrium neutrino oscillations in the early universe with an inverted neutrino mass hierarchy," Phys. Lett. B 385, 159 (1996) hep-ph/9610399.

[10] J. Pantaleone, "Stability of incoherence in an isotropic gas of oscillating neutrinos," Phys. Rev. D 58, 073002 (1998).

[11] R. F. Sawyer, "Speed-up of neutrino transformations in a supernova environment," Phys. Rev. D 72, 045003 (2005) hep-ph/0503013.

[12] S. Pastor, G. G. Raffelt and D. V. Semikoz, "Physics of synchronized neutrino oscillations caused by self-interactions," Phys. Rev. D 65, 053011 (2002) hep-ph/0109035.

[13] C. Lunardini and A. Y. Smirnov, "High-energy neutrino conversion and the lepton asymmetry in the universe," Phys. Rev. D 64, 073006 (2001) hep-ph/0012056.

[14] A. D. Dolgov, S. H. Hansen, S. Pastor, S. T. Petcov, G. G. Raffelt and D. V. Semikoz, "Cosmological bounds on neutrino degeneracy improved by flavor oscillations," Nucl. Phys. B 632, 363 (2002) hep-ph/0201287.

[15] Y. Y. Y. Wong, "Analytical treatment of neutrino asymmetry equilibration from flavour oscillations in the early universe," Phys. Rev. D 66, 025015 (2002) hep-ph/ 0203180.

[16] K. N. Abazajian, J. F. Beacom and N. F. Bell, "Stringent constraints on cosmological neutrino antineutrino asymmetries from synchronized flavor transformation," Phys. Rev. D 66, 013008 (2002) astro-ph/0203442.

[17] J. T. Pantaleone, "Neutrino flavor evolution near a supernova's core," Phys. Lett. B 342, 250 (1995) astro-ph/9405008.

[18] Y. Z. Qian and G. M. Fuller, "Neutrino-neutrino scat- tering and matter enhanced neutrino flavor transformation in Supernovae," Phys. Rev. D 51, 1479 (1995) astro-ph/9406073.

[19] G. Sigl, "Neutrino mixing constraints and supernova nucleosynthesis," Phys. Rev. D 51, 4035 (1995) astro-ph/9410094.

[20] S. Pastor and G. Raffelt, "Flavor oscillations in the supernova hot bubble region: Nonlinear effects of neutrino background," Phys. Rev. Lett. 89, 191101 (2002) astro-ph/0207281.

[21] A. B. Balantekin and H. Yüksel, "Neutrino mixing and nucleosynthesis in core-collapse supernovae," New J. Phys. 7, 51 (2005) astro-ph/0411159.

[22] G. M. Fuller and Y. Z. Qian, "Simultaneous flavor transformation of neutrinos and antineutrinos with dominant potentials from neutrino neutrino forward scattering," Phys. Rev. D 73, 023004 (2006) astro-ph/0505240.

[23] H. Duan, G. M. Fuller and Y. Z. Qian, "Collective neutrino flavor transformation in supernovae," astro-ph/0511275

[24] H. Duan, G. M. Fuller, J. Carlson and Y. Z. Qian, "Simulation of coherent non-linear neutrino flavor transformation in the supernova environment. I: Correlated neutrino trajectories," Phys. Rev. D 74, 105014 (2006) astro-ph/0606616.

[25] H. Duan, G. M. Fuller, J. Carlson and Y. Z. Qian, "Coherent development of neutrino flavor in the supernova environment," astro-ph/0608050.

[26] S. Hannestad, G. G. Raffelt, G. Sigl and Y. Y. Y. Wong, "Self-induced conversion in dense neutrino gases: Pendulum in flavour space," Phys. Rev. D 74, 105010 (2006) astro-ph/0608695.

[27] A. Friedland and C. Lunardini, "Neutrino flavor conversion in a neutrino background: Single- versus multiparticle description," Phys. Rev. D 68, 013007 (2003) hep-ph/0304055.

[28] A. Friedland and C. Lunardini, "Do many-particle neutrino interactions cause a novel coherent effect?," JHEP 0310, 043 (2003) hep-ph/0307140.

[29] A. Friedland, B. H. J. McKellar and I. Okuniewicz, "Construction and analysis of a simplified many-body neutrino model," Phys. Rev. D 73, 093002 (2006) hep-ph/0602016.

[30] A. D. Dolgov, "Neutrinos in the early universe," Yad. Fiz. 33, 1309 (1981) [Sov. J. Nucl. Phys. 33, 700 (1981)].

[31] M. A. Rudzsky, "Kinetic equations for neutrino spin- and type-oscillations in a medium," Astrophys. Space Science 165, 65 (1990).

[32] G. Sigl and G. Raffelt, "General kinetic description of relativistic mixed neutrinos," Nucl. Phys. B 406, 423 (1993).

[33] B. H. J. McKellar and M. J. Thomson, "Oscillating doublet neutrinos in the early universe," Phys. Rev. D 49, 2710 (1994). 